\title{
MITO: a new directional muon telescope
}

\author{
Sindulfo Ayuso ${ }^{1,2, *}$, Juan José Blanco ${ }^{1,2}$, Juan Ignacio García Tejedor ${ }^{1,3}$, Raúl Gómez Herrero ${ }^{1,2}$, \\ Iván Vrublevskyy ${ }^{1}$, Óscar García Población ${ }^{1,3}$, and José Medina ${ }^{2}$ \\ ${ }^{1}$ Castilla-La Mancha Neutron Monitor, Space Research Group, Parque Científico y Tecnológico de Castilla-La Mancha, \\ Avda. Buendía, 11, 19005 Guadalajara, Spain \\ 2 Department of Physics and Mathematics, Space Research Group, Universidad de Alcalá, Ctra. Madrid-Barcelona km 33,6, \\ 28871 Alcalá de Henares, Spain \\ ${ }^{3}$ Department of Computing Engineering, Space Research Group, Universidad de Alcalá, Ctra. Madrid-Barcelona km 33,6, \\ 28871 Alcalá de Henares, Spain
}

Received 1 June 2020 / Accepted 24 December 2020

\begin{abstract}
Muon telescopes are instruments devoted to the observation of muons. They are produced in the atmosphere by means of the interaction of cosmic ray and solar energetic particles with atmospheric nuclei. Muons, as cosmic rays that produce them, present non uniform arrival directions and temporal variations at ground level and, along certain observation directions, could forecast the arrival of interplanetary coronal mass ejections (ICMEs) at the Earth, even earlier than neutron monitors. However, multidirectional muon telescopes are not easily affordable because of their complexity, size and cost. In this work, we present the muon impact tracer and observer (MITO) design concept. It is composed of only two stacked scintillators $\left(1 \mathrm{~m}^{2}\right)$ with an optional lead layer that allows the filtering of unwanted particles depending on the type of application. In the case presented here, a $10 \mathrm{~cm}$ lead layer corresponding to the lead of a 3NM64 neutron monitor around which MITO has been built. Eight photomultipliers (PMTs) gather the light emerging from the four lateral sides of the scintillators. MITO has been conceived not only to achieve muon flux registering, but also to register muon arrival directions through the capture and analysis of multiple PMT pulse height data. The number of scintillators and electronic components is reduced, simplifying its design and construction and reducing complexity, volume, weight, power consumption and cost, and thus, achieving a reasonable performance-cost ratio in comparison to other directional telescopes based on two-layer matrices. The first prototype was shipped from Spain to Antarctica where it is now recording data. Some preliminary results are also presented.
\end{abstract}

Keywords: cosmic ray / instrumentation / muon telesope

\section{Introduction}

Cosmic rays (CR) are energetic particles that constantly hit the Earth's atmosphere, being source of a background ionizing radiation. As these particles travel the heliosphere, they are modulated by different disturbances generated at the Sun, like coronal mass ejections (CMEs) and shock waves (Cane et al., 2000; Blanco et al., 2013a). CR are of great interest from a space-weather perspective for several reasons. First, they travel at nearly the speed of light, much faster than solar disturbances like shocks or CMEs, making it possible to provide advance warning of a disturbance approaching Earth. Second, they have large mean free paths of the pitch-angle scattering. This is important because precursory signatures of an approaching disturbance are wiped out by the scattering after the particles

\footnotetext{
*Corresponding author: sindulfo. ayuso@edu. uah. es
}

have traveled beyond a mean free path. Thirdly, the Larmor radii of cosmic rays in the interplanetary magnetic field (IMF) are larger than the size of Earth's magnetosphere, but smaller than the typical scale size of disturbances. The energy of cosmic rays at which ground based muon telescopes have significant response is from approximately $10 \mathrm{GeV}$ to several hundred $\mathrm{GeV}$, being the highest about $50 \mathrm{GeV}$. This range of $10-100 \mathrm{GeV}$ corresponds to a Larmor radii of 0.04 0.44 AU when assuming an interplanetary magnetic field of $5 \mathrm{nT}$. This is significant because it implies that the kinetic anisotropy and gradient of arriving secondary muons mainly reflect the structure of the disturbance of this scale range (da Silva et al., 2004).

Most of the CR energy reaches the Earth's surface in the form of kinetic energy of relativistic muons, which are secondary products of interactions between highly energetic CR and nuclei of atmospheric particles (Cecchini \& Spurio, 2012). 
Muons $(\mu-$ and $\mu+)$ are particles belonging to the lepton family and they have the same charge (negative and positive, respectively) as that of an electron and 207 times its mass. Due to its electric charge, muons can be easily detected by means of scintillators, being the most numerous charged particles at sea level. The mean energy of muons at ground is $\approx 4 \mathrm{GeV}$ and their overall angular distribution (muons $\mathrm{m}^{-2} \mathrm{sr}^{-1}$ ) as a function of zenith angle $\theta$ is $\approx \cos ^{2} \theta$ for those with energy $E_{\mu} \approx 3 \mathrm{GeV}$ (Tanabashi et al., 2018).

Both neutron monitors and muon telescopes are very useful in the space weather forecasting, either by analyzing the count rate or by studying anisotropies obtained with directional muon telescopes. Therefore, the data provided by the Neutron Monitor Data Base (NMDB) and the Global Muon Detector Network (GMDN) are really helpful in space weather forecasts (Mavromichalaki et al., 2004; Rockenbach et al., 2014). Muon observations are complementary to neutron monitor studies. Neutron monitor observations extend from the lowest energies, accessible to ground based observation, up to approximately $50 \mathrm{GeV}$, while muon observations have significant responses from approximately $10 \mathrm{GeV}$ to several hundred $\mathrm{GeV}$ (Duldig, 2000).

Variations, both in the neutron monitors and muon telescopes counting rates, can be proxies of changes in the propagation conditions of cosmic rays through the heliosphere. For instance, the depth and duration of decreases in counting rates known as Forbush decreases can be related to the velocity of ICMEs and driven shocks, the intensity of their magnetic field and deceleration of ICME (Blanco et al., 2013a, b).

An important aspect of space weather prediction is the determination of whether or not an interplanetary shock is headed toward the Earth and at approximately what time its impact is expected. While the relationship between coronal mass ejections (CMEs) and Forbush decreases in Galactic cosmic rays is now well established (Cane, 2000), it is less generally recognized that cosmic-ray decreases are often accompanied by strong enhancements of the cosmic-ray anisotropy. On the other hand, ground-based observations of cosmic rays by neutron monitors and muon detectors have found precursory anisotropies, loss cone precursors (Munakata et al., 2005), before the arrival of an interplanetary shock and subsequent Forbush decrease, possibly providing an advance warning of space weather effects on shock impact at the Earth's magnetosphere. Surprisingly, muon detectors observe precursors earlier than neutron monitors (Leerungnavarat et al., 2003). This is due to the fact that muon detectors respond to higher energy cosmic rays (greater than $50 \mathrm{GeV}$ ) than neutron monitors (about $10 \mathrm{GeV}$ ) (Munakata et al., 2000).

Muon tracking has traditionally been performed using coincidence in multiple detectors laid out in a two layer matrix with a lead layer between them to filter out lower energy particles. For instance, the Nagoya (Japan), Hobart (Australia) and Sao Martinho da Serra (Brasil) multi-directional muon telescopes are similar (with layers separated $1.73 \mathrm{~m}$ ) except for the number of individual detectors $\left(1 \mathrm{~m}^{2}\right.$ scintillators $)$ on each horizontal layer. There are $6 \times 6$ in the first, $4 \times 4$ in the second and $4 \times 9$ individual detectors in the third. On the other hand, the Kuwait telescope is composed of four $5 \times 5 \mathrm{~m}$ layers (0.8 $\mathrm{m}$ apart) of cylindrical proportional counter tubes below a lead layer (Mendonça et al., 2019). When a coincidence is registered between two detectors, one at each layer, the trajectory of a particle can be determined. Due to simple geometric reasons, the angular resolution depends on the size of each individual detector and the distance between layers. Thus, the angular resolution increases as the size of detectors decreases and/or the distance between layers increases. On the other hand, traditional muon telescopes have large sizes in order to obtain high statistics allowing the recording of small variations in muon flux. Table 1 shows a comparison between MITO and other directional muon telescopes in terms of main elements used: volume, angle of view, detection area and counting rate.

Regarding the techniques used for the detection of coincidences in most of multidirectional telescopes, both hardware and software approaches (Ayuso et al., 2016) are widely used with different purposes such as reducing noise; getting directional information (Karapetyan et al., 2013); reducing the probability of a measurement being triggered by independent, unrelated particles; lessening the probability of independent random background events or identifying energetic particles in multi-element particle telescopes (Müller-Mellin et al., 1995).

Traditional particle detection systems make use of dedicated hardware to process data and get instant information. When real-time operation is not required, alternative approaches based on the analysis of recorded pulse information can be used (Havelka et al., 2002). These systems are based on the registration of pulse properties (e.g. amplitude voltages) and their corresponding accurate timestamps. The recorded data are then processed by software in order to obtain the coincidence counting rates, particles trajectory and any other relevant information.

In this work, we present MITO (muon impact and trace observer), an affordable directional muon telescope, made up of two $1 \mathrm{~m}^{2}$ scintillators, which is able to provide counting rates. Furthermore, it uses coincidence techniques to identify the passage of each muon through the scintillators, pulse height comparison techniques to determine the point of impact in each scintillator and, from them, the particle trajectory. This muon telescope is a complement to neutron monitors, being able to obtain anisotropies of muon flux and, therefore, anticipating the arrival of certain solar events on Earth. The main purpose of this paper is to illustrate the instrument concept and its capabilities. We are currently working in the characterization of the instrument response for different incident directions in order to derive quantitative anisotropy data, although this goes beyond the scope of the present paper.

\section{Telescope implementation}

MITO is part of ORCA ("Observatorio de Rayos Cósmicos Antártico", in English it would be "Antarctic Cosmic Ray Observatory"), which has been recently deployed by the University of Alcalá at the Juan Carlos I Antarctic Spanish Base in Livingston Island (Antarctic Peninsula) in January 2019 (Ayuso et al., 2019; Blanco et al., 2019). ORCA is a combination of a NEutron MOnitor (NEMO), which is a direct heritage of CaLMa (CAstilla-La MAncha Neutron Monitor) (Medina et al., 2013) and MITO, a muon telescope which shares with NEMO the same stack and housing. ORCA's main objectives are to measure the flux of CRs in a region not covered by the NMDB and to study solar activity, which can be inferred from CR flux temporal variations. 
Table 1. Comparison between MITO and other directional muon telescopes. In Kuwait, a different approach is used, using four layers of proportional counter tubes (PCT) instead of scintillators. The viewing angle and maximum zenith angle have not been taken from the official specifications of each telescope, but they are approximate values obtained by calculation from each telescope dimensions in order to make a rough comparison between MITO and other telescopes. The value of the maximum zenith angle corresponds to the vertical plane of the diagonal. Detection areas and counting rates have been taken from (Munakata et al., 2018).

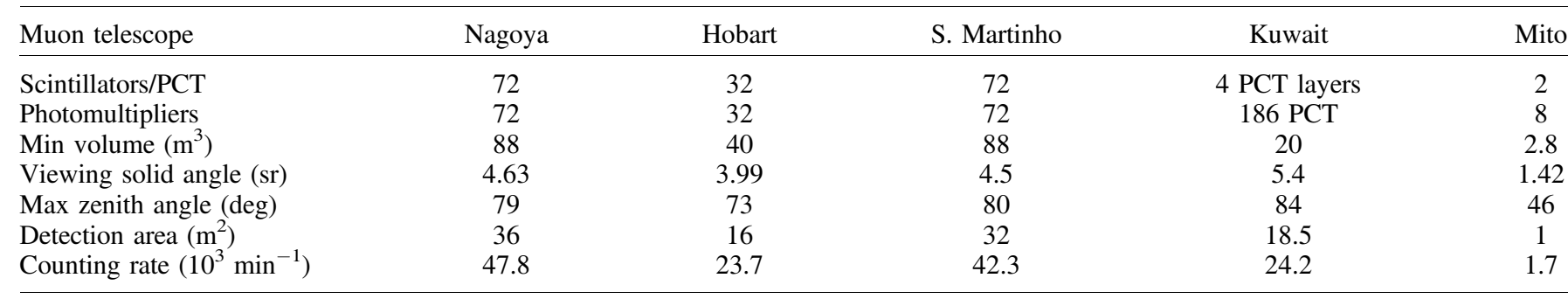

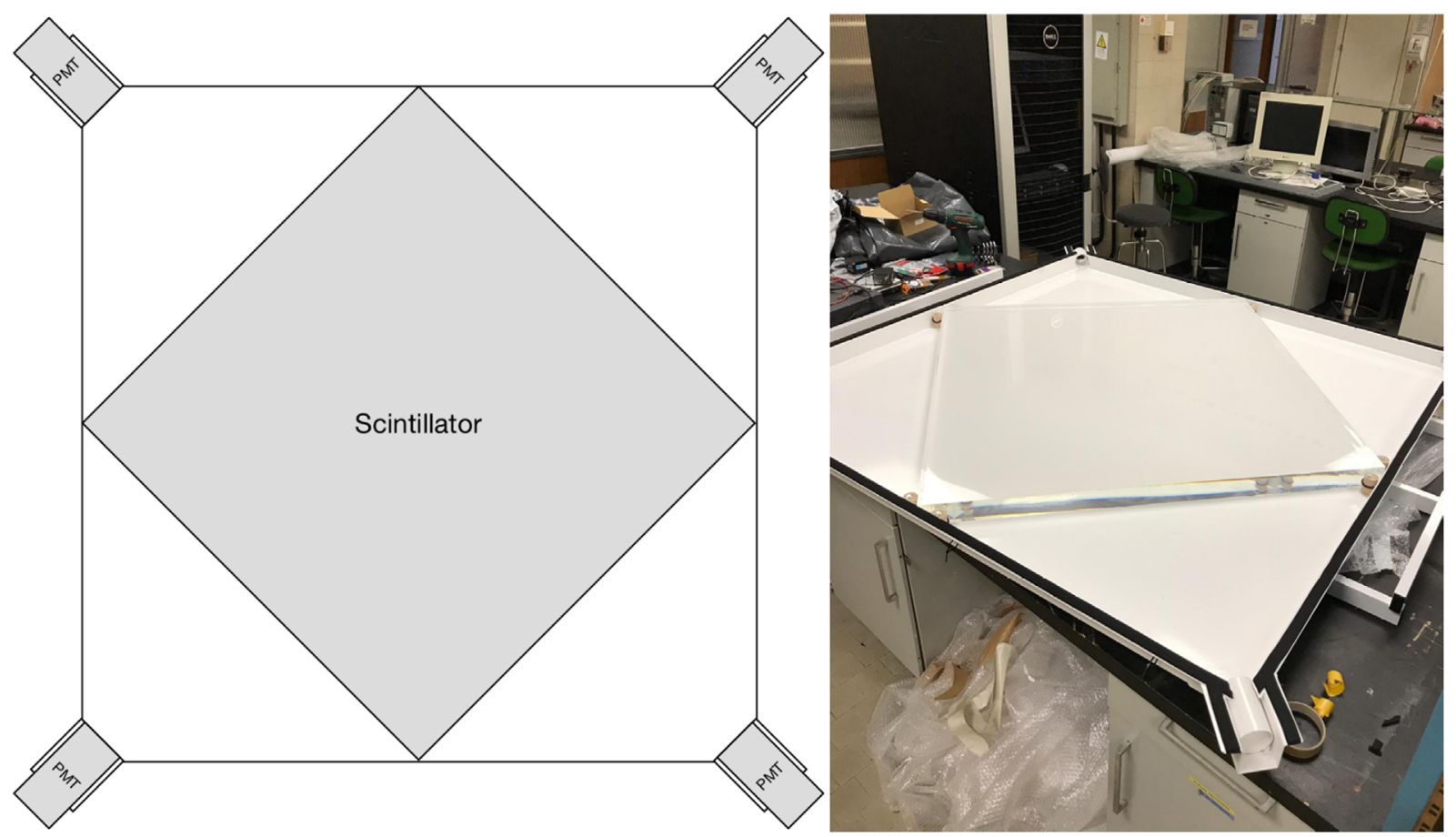

Fig. 1. On the left, scintillator, PMTs and pyramidal light guides configuration in each MITO device. On the right, scintillator inside the enclosure, which also serves as a light guide.

ORCA has been implemented inside a 20 feet standard marine cargo container whose interior walls have been covered with $10 \mathrm{~cm}$ of polyurethane foam to insulate the electronic devices inside from external temperature variations. MITO is composed of two identical devices (MITO-Top and MITOBottom), one stacked above the other $136.5 \mathrm{~cm}$ apart, each consisting of a BC-400 organic scintillator $(100 \mathrm{~cm} \times$ $100 \mathrm{~cm} \times 5 \mathrm{~cm}$, poly-vinyl-toluene with $65 \%$ anthracene) and four PMTs (Hamamatsu R2154-02) laid out around the four narrow sides of the scintillator, pointing inwards at a distance of $50 \mathrm{~cm}$. All those elements have been installed inside an aluminum housing, which includes four triangular prism-shaped light guides closed in such a way that prevents the passage of light from the outside (Fig. 1). The surface of the guide walls has been painted in matte white in order to get Lambertian reflection. Lambertian reflectance models a perfect diffuse surface that scatters incident illumination equally in all directions. It is a reasonable approximation to many real-world surfaces such as matte paint (Pharr et al., 2017).

Both MITO devices, MITO-Top and MITO-Bottom, have been mounted in the ORCA structure (Fig. 2) with a $10 \mathrm{~cm}$ thick lead layer between them belonging to the neutron monitor. This lead layer works as a filter, avoiding the passage of charged particles below $200 \mathrm{MeV}$ in the case of muons, $160 \mathrm{MeV}$ for protons and more than $5 \mathrm{GeV}$ for electrons acording to our simulations based on GEANT4 (see Sect. 2).

\subsection{Viewing angle}

A viewing angle given by the expression $\alpha=2 \arctan (d / h)$ is obtained based on the dimensions of the scintillators and the separation between them (Fig. 3). According to this, the viewing 

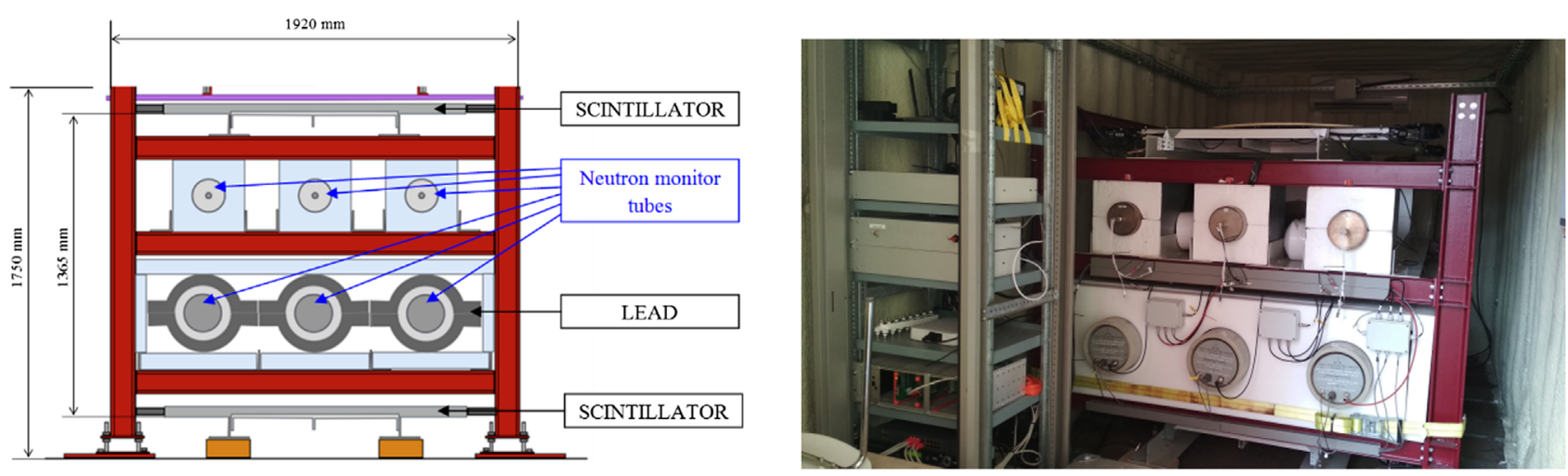

Fig. 2. Layout of the final setup and its installation into the ORCA marine container.

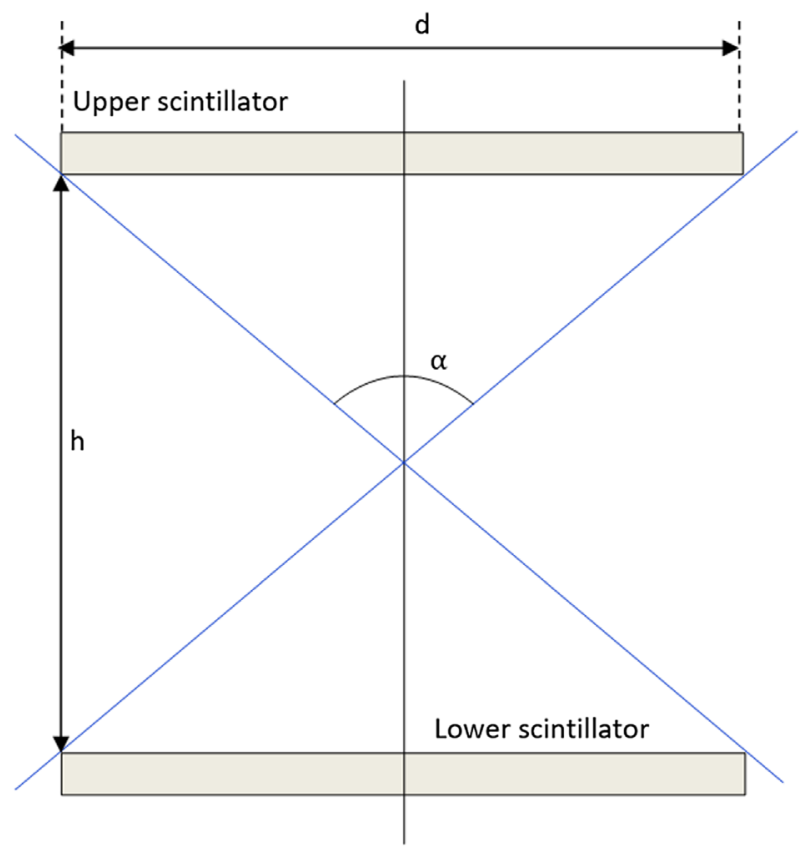

Fig. 3. Illustrative diagram of the viewing angle $(\alpha=2 \arctan (d / h))$, where $h=1.365 \mathrm{~m}$ in MITO. Therefore, $\alpha$ varies between $72.4^{\circ}$ for the vertical plane parallel to the lateral sides of the scintillator $(d=1 \mathrm{~m})$ and $92^{\circ}$ for the vertical plane containing the diagonals $(d=\sqrt{2} \mathrm{~m})$. The solid angle for the whole set equals $1.42 \mathrm{sr}$.

angle varies between $72.4^{\circ}$ when the direction from side to side of each scintillator is considered $(d=1 \mathrm{~m}, h=1.365 \mathrm{~m})$, and $92^{\circ}$ in the diagonal direction, from corner to corner $(d=1.41 \mathrm{~m}, h=1.365 \mathrm{~m})$. This gives a solid angle of 1.42 sr. This value is lower than those for conventional Nagoya-type telescopes, as can be seen in Table 1 .

\section{Theory of operation}

The light generated by a charged particle passing through the scintillator comes out through the four narrow lateral faces of the prism, and is led by the light guides towards the four photomultipliers (Fig. 4). Each one collects the light and generates a pulse whose amplitude carries information, both about the deposited energy and the distance between the impact point and the corresponding lateral surface nearest to the PMT. The impact point coordinates in every scintillator can be determined by comparing the signal level of the pulses generated by opposite PMTs.

Once the two impact points are determined, the muon incident direction can be computed (left picture in Fig. 5).

\subsection{GEANT4 simulation.}

The purpose of this simulation is to build a software model of the telescope with identical characteristics to the real one using the GEANT4 tool developed by CERN, which is a toolkit for the simulation of the passage of particles through matter. The objective is to reproduce a flux of particles (mainly muons) in an isotropic and random way. When generating the trajectories, the impact coordinates in MITO-Top and the direction of the trajectory of each particle are known. The rest of the data (such as the number of photons generated in the scintillator, the energy losses when passing through them and through the lead, or the number of photons that leave the scintillator through each lateral face) are obtained as a result of the simulation. Since the current generated by a PMT due to the passage of each particle is a function of the number of photons that reach the PMT (cathode luminous sensitivity in $\mu \mathrm{A} / \mathrm{lm}$ ), a pulse whose amplitude is proportional to the number of photons hitting the cathode is generated by the PMT.

\subsubsection{Simulation characteristics}

The simulation geometry is composed of two identical scintillators and the $10 \mathrm{~cm}$ lead layer between them, which allows us to simulate impact on the primary particle passage (Fig. 5 right plot). The GEANT4 version of MITO is built of a hollow box, which corresponds to the aluminum container with the scintillator placed inside. The corners of the box were cut off to allow photons to pass to the PMT zone, where each photon is registered and analyzed at the end of the event. Moreover, four sensitive detectors were placed near the scintillator to analyze how many photons go out through each face of the scintillator and compare it with the amount of them which are absorbed inside the box. Along with a sensitive detector for photons, there is one detector inside the lead layer and another one inside each scintillator, whose purpose is to measure energy 

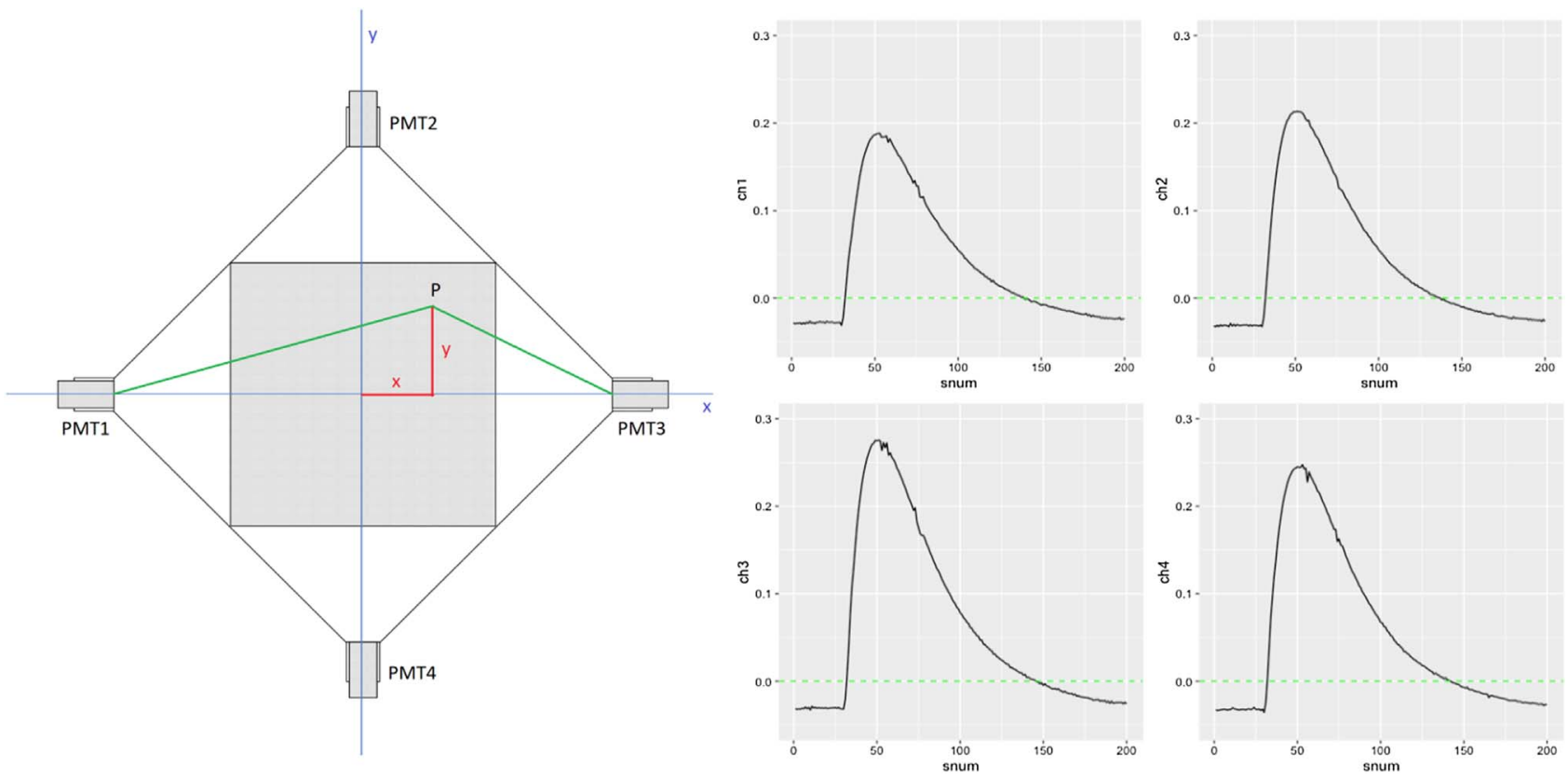

Fig. 4. On the left, axis and coordinates of a point. On the right, pulses captured by the PMTs in coincidence. The $x$ coordinate is calculated applying an algorithm from the pulse height of PMT1 and PMT3. In the same way, the $y$ coordinate is obtained from PMT4 and PMT2 signals.

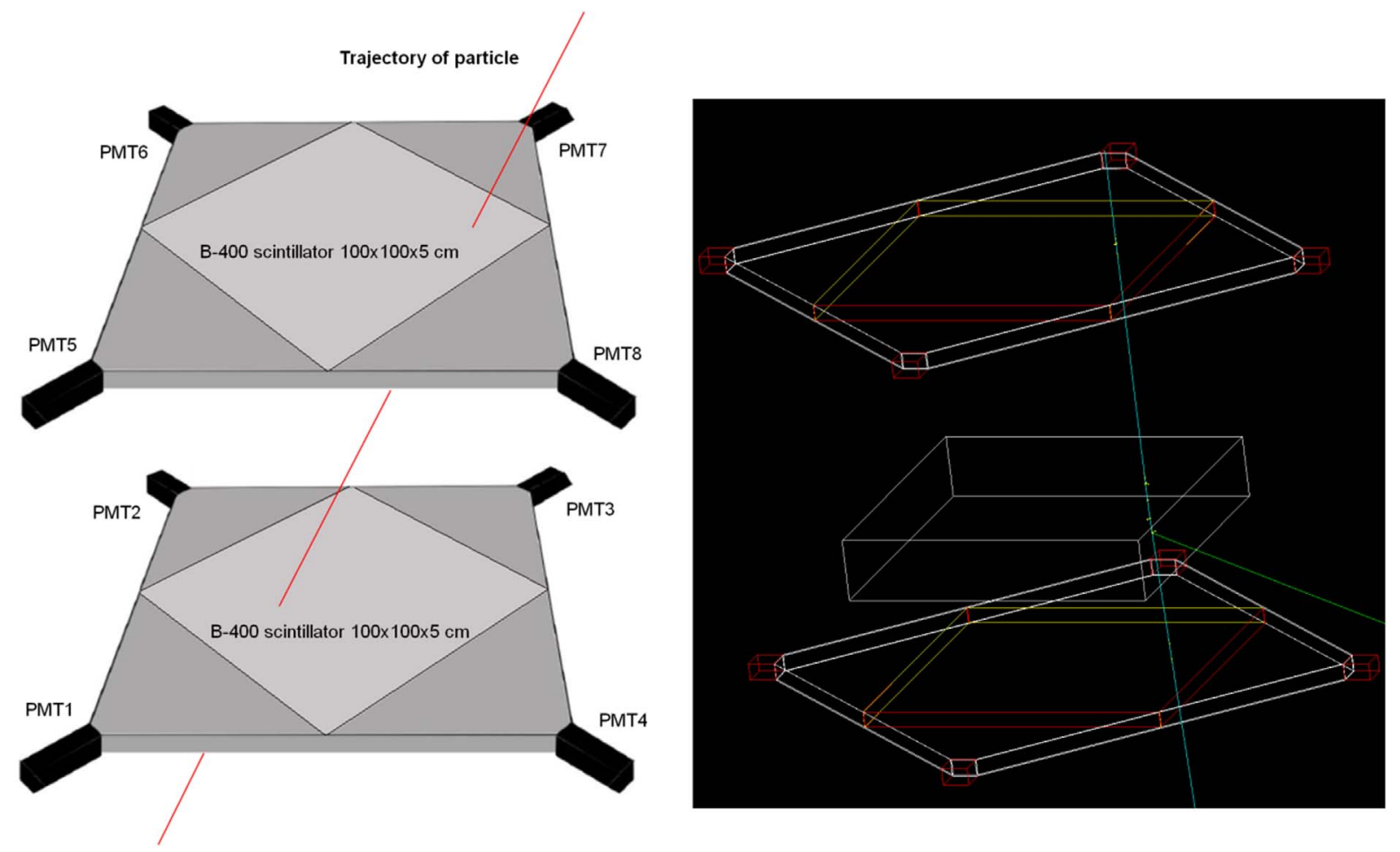

Fig. 5. On the left, cartoon showing the muon trajectory throughout MITO. On the right, GEANT 4 image representing the MITO elements included in the simulation: Aluminum hollow box with light guides, scintillators and lead layer.

loss of the primary particle inside each volume and record track information which is analyzed at the end of the event. With the exception of the lead layer, which has been taken into account not only because it works as a shield for low energetic particles, but also because it slightly deflects the trajectory of muons, the rest of NEMO materials have a negligible influence on muon trajectories (Aguayo Navarrete et al., 2011) and they have not been taken into account in the simulation.

In order to identify muon impact points on the scintillator, we set a reference Cartesian coordinate system centred at the 

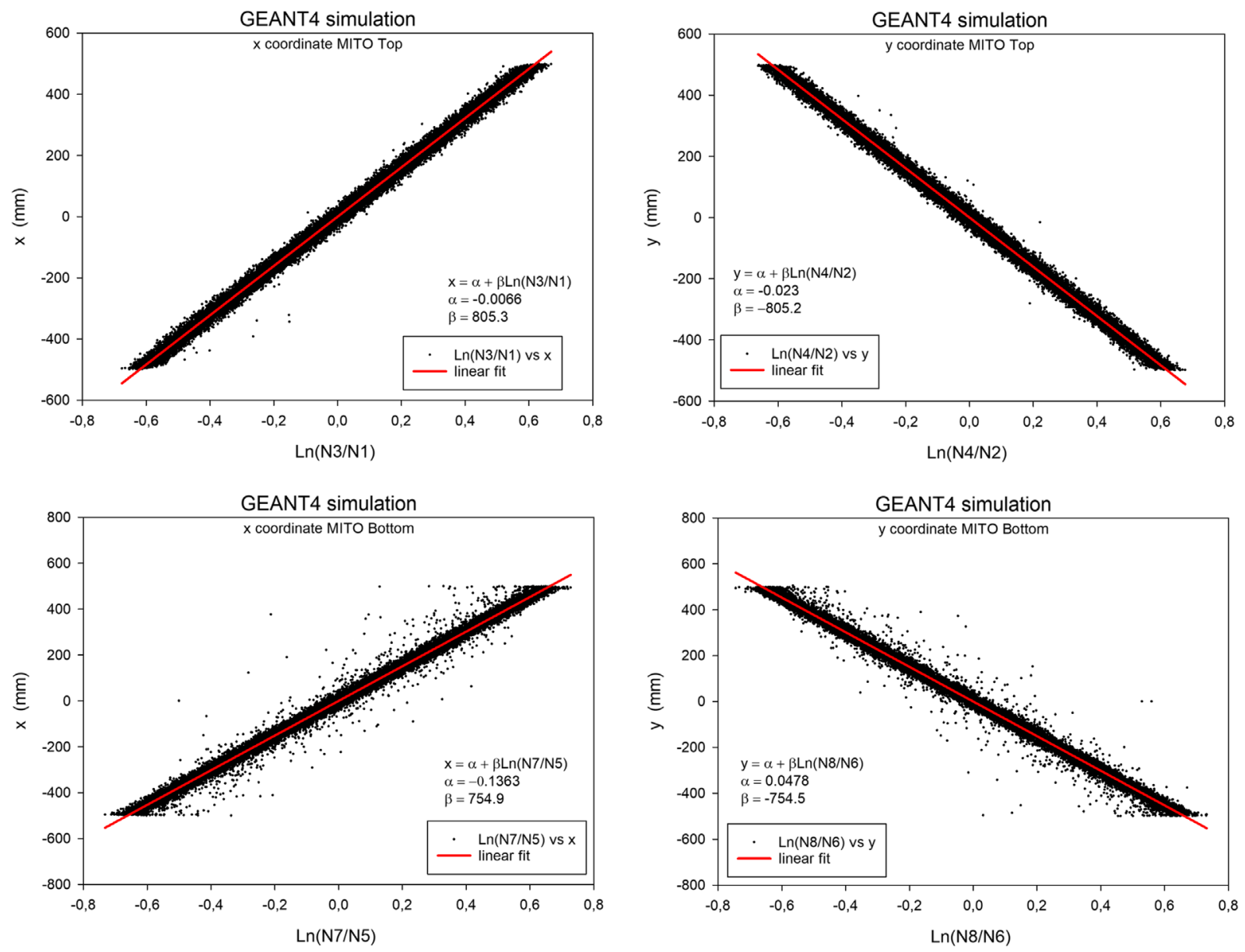

Fig. 6. Scatter plots showing the relationship between the simulated coordinate and the logarithm of the ratio between the total light emitted by opposite faces on the corresponding axis.

scintillator centre, the $x$-axis aligned with the axes of symmetry of PMT1 and PMT3, and the $y$-axis aligned with the axes of symmetry of PMT2 and PMT4 (Fig. 4).

The following physics processes were enabled for this simulation among others: reflection, refraction and absorption for photons and ionization, bremsstrahlung, scattering and pair production for muons, besides other processes for secondary particles.

The GEANT4 unified model with "groundfrontpainted" surface finish has been activated for the scintillator container aluminum surface to control Lambertian reflection and absorption processes because of a matte white paint has been applied to the light guides.

\subsubsection{Impact points coordinates}

A Monte Carlo simulation of 20,000 muons has been carried out in order to obtain an algorithm that calculates the coordinates of the muon impact point in the scintillator based on the pulse levels (number of photons) gathered by the PMTs.

The scatter plots in Figure 6 show, for each coordinate, the relationship between the impact point coordinate and the ratio between the total light emitted by the lateral sides of the scintillator that are perpendicular to the corresponding coordinate axis.
The distribution may be fitted by linear regression resulting in the following expressions for coordinates in MITO-Top $\left(x_{T}, y_{T}\right)$ and MITO-Bottom $\left(x_{B}, y_{B}\right)$ :

$$
\begin{aligned}
& x_{T}=805.3 \ln \frac{N_{3}}{N_{1}}-0.0066 \\
& y_{T}=-805.2 \ln \frac{N_{4}}{N_{2}}-0.023 \\
& x_{B}=754.9 \ln \frac{N_{7}}{N_{5}}-0.1363 \\
& y_{B}=-754.5 \ln \frac{N_{8}}{N_{6}}+0.0478
\end{aligned}
$$

where $N_{x}$ is the number of photons emitted through the scintillator face towards the PMTx light guide.

Therefore, assuming that the four PMTs gather a proportional amount of photons emerging through their respective scintillator side, both $x$ and $y$ coordinates can be directly calculated in both scintillators from equations (1)-(4).

Once the coordinates of the point of impact on both scintillators have been determined, the azimuth and zenith angles for 

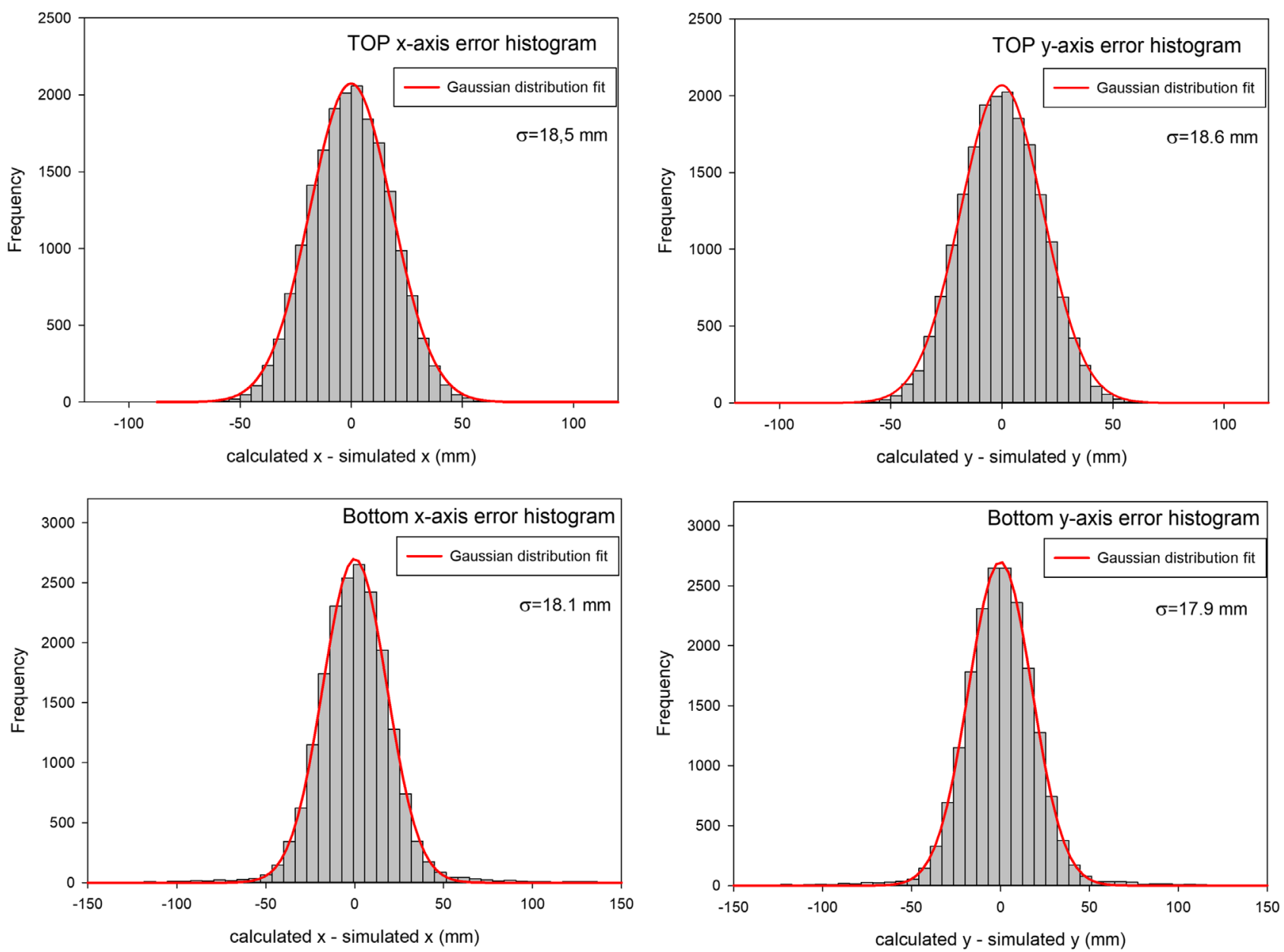

Fig. 7. Histograms with the difference between simulated coordinates and calculated coordinates (including lead layer deviation effect) fitted to a Gaussian distribution. The error $e$ in $99,7 \%$ of all calculated coordinates is lower than $e= \pm 3 \sigma= \pm 55.8 \mathrm{~mm}$.

every incident particle are computed by means of the two relationships given in the following equations:

$$
\begin{gathered}
\phi=\arctan \frac{y}{x}=\arctan \frac{y_{T}-y_{B}}{x_{T}-x_{B}} \\
\theta=\arctan \frac{\sqrt{x^{2}+y^{2}}}{z}=\arctan \frac{\sqrt{\left(x_{T}-x_{B}\right)^{2}+\left(y_{T}-y_{B}\right)^{2}}}{z}
\end{gathered}
$$

being $\phi$ the azimuth angle, $\theta$ the zenith angle, $x_{T}$ and $x_{B}$ the $x$ coordinates on top and bottom respectively, $y_{T}$ and $y_{B}$ the $y$ coordinates on top and bottom respectively and $z$ the distance between MITO-Top and MITO-Bottom.

\subsubsection{Impact point precision and angular resolution}

The accuracy in the determination of the impact point depends on many factors, such as the proportionality between the conversion of the received light and the height of the pulse, the number of reflections of the photons before reaching the photomultiplier and the uncertainty of the processes involved in the production of light and its measurement. The correct determination of the accuracy of MITO requires the use under controlled conditions of suitable radioactive sources but this has not been possible before its implementation. However, it is possible to use the simulation described in Section 3.1 to give an estimate of the precision in determining the point of impact and the direction of incidence. The simulated impact points has been compared to the calculated impact points inferred from equations (1)-(4) to fulfill this goal. Applying these formulas to calculate the $x$ and $y$ coordinates for all impact points and then obtaining the difference between calculated and simulated values, it is possible to determine the error $e$ for the calculated impact point coordinates. Figure 7 shows the histograms of this error defined as the difference between simulated and calculated values.

The histograms in Figure 7 are fitted to a Gaussian distribution, being the standard deviation $\sigma=18.6 \mathrm{~mm}$ in the worst case (y coordinate in the top scintillator). Therefore, $99.7 \%$ of the calculated coordinates will have an error $e \leq \pm 3 \sigma \equiv e \leq$ $\pm 55.8 \mathrm{~mm}$. The muon deviation passing through the lead layer has also been taken into account. According to the previous results, it can be considered that the particle has passed through some region into a $11.16 \mathrm{~cm}$ diameter circular area centered at the calculated point of impact. 
Once the error $e$ is computed, the angular resolution can be obtained by means of a geometric analysis. A drawing of the applied geometrical considerations is outlined in Figure 8. It is clear from the figure that there is a dependence between the zenith angle and the angular resolution resulting the latter increasing as the zenith angle increases. For vertical trajectories, $\theta=0$, the angular resolution is equal to $4.7^{\circ}$. It is possible make a rough comparison between MITO and Nagoya telescope applying the same geometrical considerations and taking into account Nagoya dimensions (1.73 m between matrix layers). The Nagoya zenith angular resolution will be ranged between $30(\arctan (1 / 1.73))$ and $39(\arctan (1.41 / 1.73))$ degrees, depending on the considered direction, scintillator edge $(1 \mathrm{~m})$ or scintillator diagonal $(1.41 \mathrm{~m})$. Applying the same calculation process for Kuwait telescope, with PCT of $10 \mathrm{~cm}$ diameter and $0.8 \mathrm{~m}$ between layers, it would give an angular resolution between $7^{\circ}$ and $10^{\circ}$ for vertical trajectories.

To check the validity of the geometrical approach and the comparison between MITO and Nagoya Telescope, the MITO angular resolution has also been estimated using the GEANT4 simulation and applying equations (5) and (6) to compute the estimated zenith and azimuth angles. The accuracy of the angle estimations is defined as the difference between the simulated and the calculated zenith and azimuth angles. Figure 9 shows the histograms of these errors fitted to the Gaussian distribution function. The error yielded in $99.7 \%$ of the trajectories is lower than $\pm 3 \sigma$, that is, $\pm 3.9^{\circ}$ in the zenith angle and $\pm 16.2^{\circ}$ in the azimuth angle. It should be pointed out that although the geometrical approach overestimates the error when comparing it to the error calculated in the simulation, $\left( \pm 4.7^{\circ}\right.$ vs. $\left.\pm 3.9^{\circ}\right)$ it is still valid to perform the comparison between MITO and Nagoya telescope.

As a last comment, regarding the angular resolution and the precision in the impact points, it should be noted that the main goal of MITO is not to achieve high performance in both angular and impact point precision, but rather, to detect variations in time of muon trajectories and, therefore, anisotropies in the cosmic ray flux to forecast ICMEs.

\subsubsection{Particle energy loss}

The energy threshold for MITO to detect a charged particle in its current configuration, i.e. with NEMO polyethylene and lead between MITO-Top and MITO-Bottom, depends on the energy that a particle deposits along its path through MITO.

The performed simulation allowed us to calculate the energy losses of muons, electrons and protons while passing through MITO, in order to evaluate the effect of the lead layer as a filter to suppress unwanted effects such as false coincidences, or the relative weight of electrons, protons and muons in the overall MITO counting rate. Secondary particles such as muons, electrons and protons uniformly distributed from $1 \mathrm{MeV}$ to $10 \mathrm{GeV}$ were generated and fired above MITO-Top at random coordinates and vertical incidence.

The MITO coincidence channel can detect charged particles coming throughout both scintillators. The kinetic energy losses for crossing MITO are, on average, $200 \mathrm{MeV}, 160 \mathrm{MeV}$ and more than $5 \mathrm{GeV}$ for muons, protons and electrons respectively. It has to be pointed out that polyethylene has not been included in the simulation because the expected effect on the secondary particle flux is negligible in comparison with lead effect

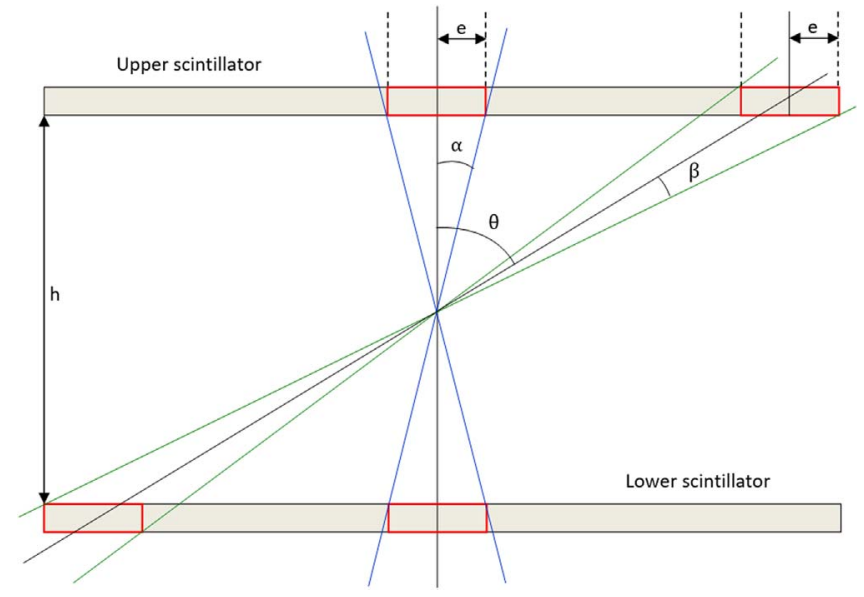

Fig. 8. Diagram of the method used for the estimation of the zenith angle resolution using a geometrical approach. $e$ is the worst case error estimation, $\theta$ is the zenith angle, $\alpha$ and $\beta$ are the angular resolution for the two extreme cases.

(Aguayo Navarrete et al., 2011). The result is summarized in Figure 10. Top panel shows the deposited energy on MITO-Top and Bottom panel shows MITO-Bottom. The $0-1 \mathrm{MeV}$ bar in the bottom panel represents stopped particles at the lead layer between MITO-Top and MITO-Bottom. None of the simulated electrons were able to impact on MITO-Bottom. Taking into account the expected energy spectra for these particles at MITO location as it is inferred from PARMA model (Sato et al., 2008), the amount of electrons crossing MITO is negligible while the number of muons is about 100 times more numerous than protons.

\section{Electronic modules}

The telescope has been equipped with two data acquisition modules that carry out similar functions using different technologies, SAS (analog processing module) and ARACNE (digital processing module). Thus, the eight analog signals from the PMTs are first amplified and then fed to both systems in parallel by means of an analog splitter system (Fig. 11), so that results from both systems can be compared and validated against each other.

\subsection{Analog processing module (SAS)}

The SAS module performs analog processing on the pulses coming from each PMT, discriminating pulses above and below certain threshold levels and, therefore, limiting the energetic particle range to process. These threshold levels are adjustable for each channel, and coincidence detection can also be configured for any arbitrary combination of channels among the eight available.

The SAS module has a triple mission:

Firstly, the adaptation of the input signal level to the allowed dynamic operating range, which is between 0 and $5 \mathrm{~V}$ peak (Fig. 12). For this, it has two adjustments: on the one hand, an attenuator and, on the other hand, a variable gain operational 

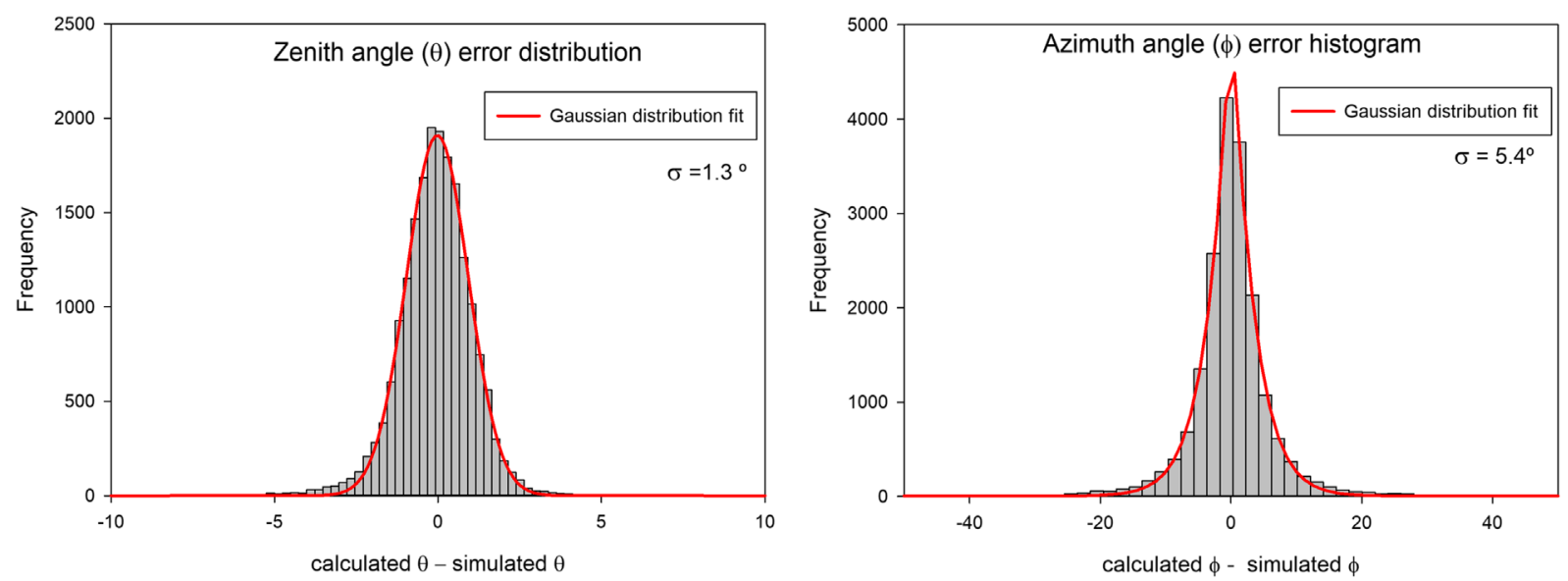

Fig. 9. Gaussian distribution fit of zenith (on the left) and azimuth (on the right) angles errors. The error in $99.7 \%$ of all calculated angles is lower than $\pm 3.9^{\circ}( \pm 3 \sigma)$ in zenith angles and lower than $\pm 16.2^{\circ}$ in azimuth angles.

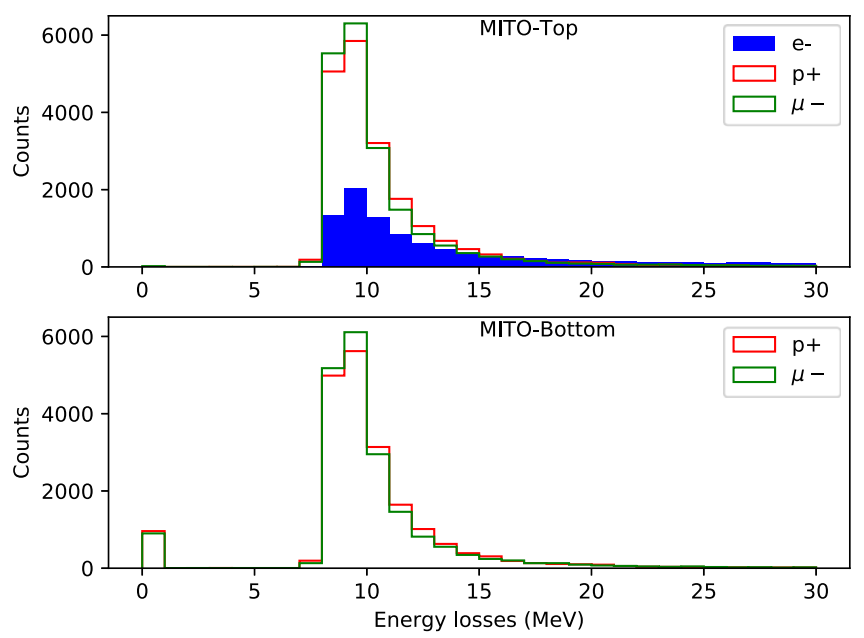

Fig. 10. Energy losses for electrons, protons and muons on MITOTop (upper panel) and on MITO-Bottom (lower panel).

amplifier that can be adjusted to increase the level of the pulses if necessary.

Secondly, the discrimination of pulses by means of a level discriminator that allows rejecting pulses exceeding a certain threshold (ULD) and therefore energy levels outside our interest, or those below a certain threshold (LLD) that correspond to noise or low energy particles.

Thirdly, the coincidence detection, by means of a module with four coincidence detectors which can be individually configured to detect a coincidence between any combination of the eight PMT channels (Fig. 13).

In order to obtain a faster temporal response, software coincidence (Ayuso et al., 2016) is not implemented, but hardware coincidence. Each coincidence detector is basically an AND gate, which allows the adjustment of a coincidence resolving time window per channel. This time window is necessary to compensate for the different propagation delays of the pulses since they are generated by each PMT until they are compared in the coincidence detector. The smaller this window is, the lower the probability of false coincidence detection.
In our case the time windows are set to $300 \mathrm{~ns}$. Bearing in mind that the accidental rate Duldig (2000), $N_{A}$, is simply:

$$
N_{A}=2 N_{1} N_{2} \tau
$$

where $N_{1}$ and $N_{2}$ are the background rate in each tray and $\tau$ is the coincidence resolving time. In our case, after adjusting the system at its current location, mean $N_{1}=188.8$ counts/s, mean $N_{2}=128$ counts/s, mean in the coincidence mode Coin8: 28.4 counts/s and $\tau=300 \mathrm{~ns}$. Thus, the value of the accidental rate $N_{A}$, is $14.4 \times 10^{-3}$ counts/s, which is $0.05 \%$ with all PMTs working in coincidence.

The output pulses generated by the four coincidence detectors are counted and registered along with a time-stamp by means of a low cost Beaglebone Black single-board computer (SBC) running a specific software. In addition, this SBC provides other capabilities such as data transmission and remote monitoring and control, which is useful if it is located in regions difficult to reach, such as Antarctica in this case. Taking into account the processing and recording times of the SBC according to the laboratory tests carried out with a pulse generator, MITO is able to detect and record a count rate of up to 100,000 pulses per minute, ten times more than the expected maximum of $\approx 10,000$ pulses per minute in the upper scintillator.

\subsection{Digital processing module (ARACNE)}

The ARACNE module (Fig. 14) works by doing all the discrimination, pulse detection and pulse height analysis digitally. After a simple analog conditioning stage, all eight signals from the PMTs are sampled in parallel using a high-speed and high-resolution ADC, and a FPGA device monitors all channels simultaneously to detect a pulse on any channel (trigger level and trigger channel(s), among other things, are software configurable).

When a pulse is detected, a window of samples of all eight channels, before and after the pulse, is captured and transferred to a SBC (also a Beaglebone Black), where coincidence and height of pulses on all channels is determined and stored to calculate points of impact on each scintillator as well as muon trajectories. By operating this way, the ARACNE module 


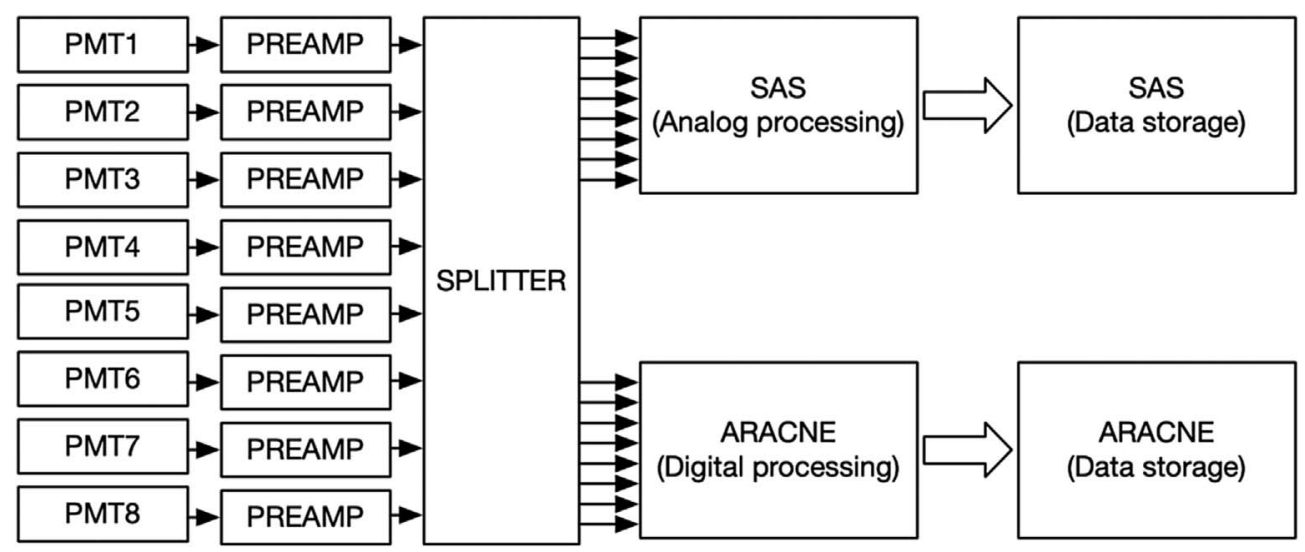

Fig. 11. MITO block diagram. The same analog signals detected by PMTs reach both process modules (analogue and digital). In this way, both results can be compared.

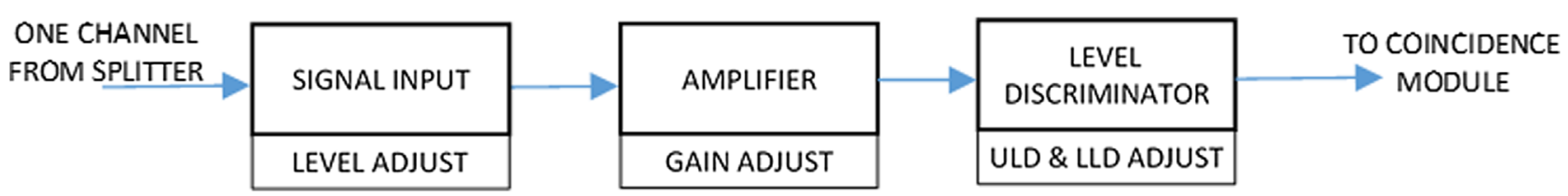

Fig. 12. Block diagram of signal analogue processing for each of the 8 input channels.

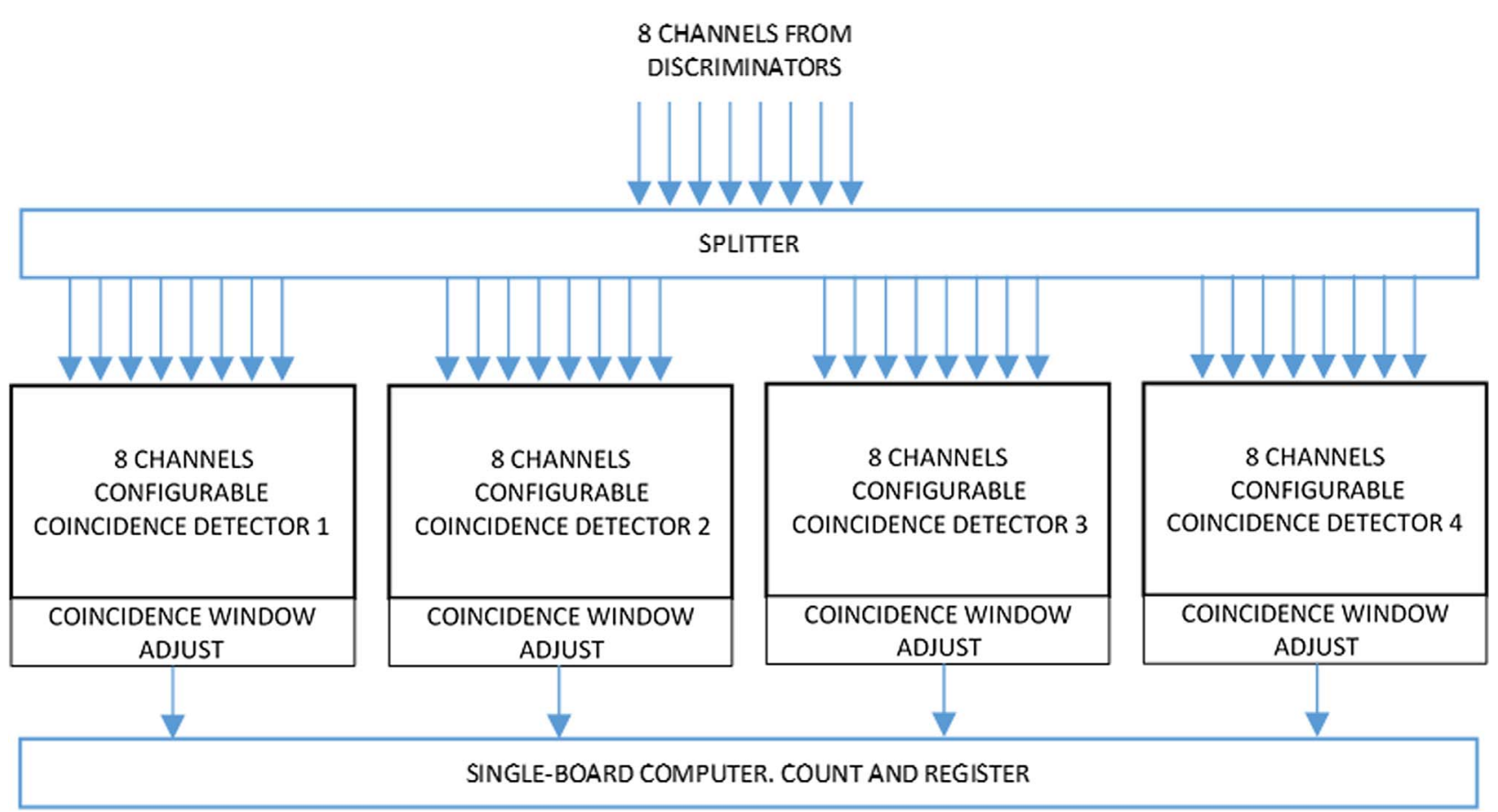

Fig. 13. Coincidence module. There are 4 identical configurable coincidence detectors. Any combination of the eight channels can be selected by software on each detector independently.

allows pulse counting as well as pulse height (or even shape) analysis, and other kinds of digital processing on the FPGA deemed necessary could be implemented.

A more detailed description of the ARACNE platform is foreseen in a future paper.

\subsection{Calibration and adjustment}

The size of MITO makes any direct calibration by positioning a radioactive source over the detector difficult. An alternative for impact point calibration could be to use cosmic rays, 

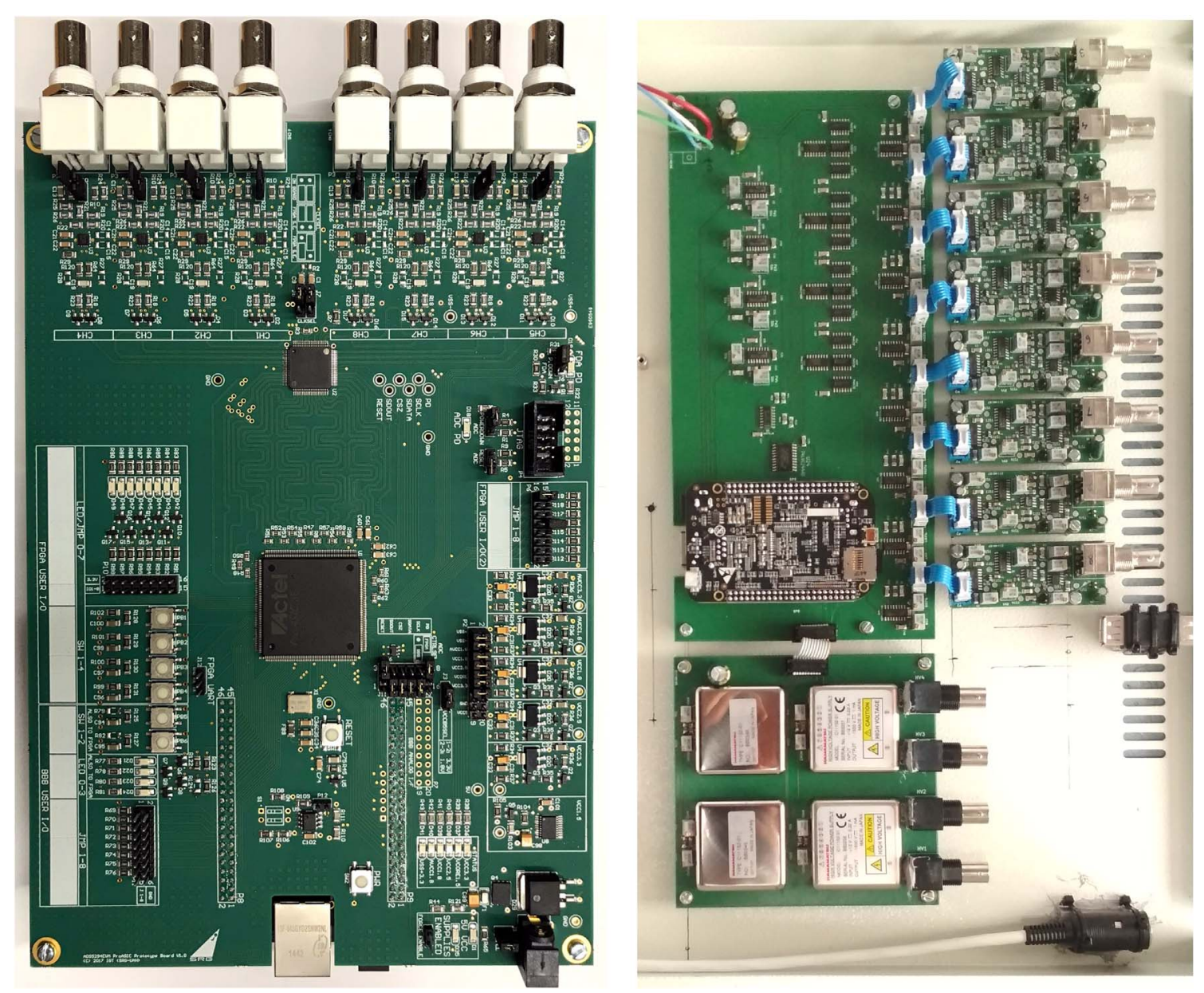

Fig. 14. ARACNE module on the left and SAS module with the HV power supply card on the right.

selecting a narrow beam by using an additional small area sensor, positioning it at specific points of the detector and using coincidence between MITO and this additional detector. Both techniques are currently under evaluation. Additionally, a novel self-organizative map technique which corrects slight misalignments during operation has been recently presented as an alternative method to a traditional calibration of MITO, but it should still be checked with a traditional calibration (Regadío et al., 2020).

Finally, the adopted calibration method is based on the signals registered by the instrument itself, adjusting the eight signal processing chains so that the level of gain and pulse discrimination results in identical pulse heights when the passage of particles through the scintillators happens at the same distance with respect to each pair of PMTs facing each other. The calibration procedure consists of two steps. First, setting the bias of each PMT, and second, setting each PMT signal level to be equal to the others by means of its statistical response after evaluation of a few thousand pulses.

\subsubsection{PMTs bias adjustment}

Due to the delicate manufacturing process of PMTs, each unit differs in their characteristics, especially regarding their cathode and anode sensitivity, which implies different gains and different high voltage (HV) bias values (Wright, 2017). Therefore, counting tests were carried out with identical gains in each channel, varying the HV bias voltage and writing down the counts of each PMT. The results are presented in Figure 15. As the system has 4 voltage sources for the 8 PMTs, they were biased in pairs with plateaus at similar voltage levels as indicated in Table 2. As it can be seen, the HV bias level was adjusted slightly above the plateau. In this way, it is also possible to detect muons with the lowest energy losses (mixed with other noise particles) when passing through the scintillator. Thus, MITO detects more noise pulses, but they are later removed by the coincidence detector, leaving a more complete muon histogram. Each of these voltages can be remotely monitored or recorded in order to verify the proper system operation.

\subsubsection{Gain and level discriminator adjustment}

Gain, upper level discriminator (ULD) and lower level discriminator (LLD) adjustments have to be carried out in both analog and digital electronic modules (SAS and ARACNE).

\section{- Analog processing module adjustment}

In the analog module there are dedicated circuits for LLD, ULD and gain signal adjustments. SAS gain adjustments were 


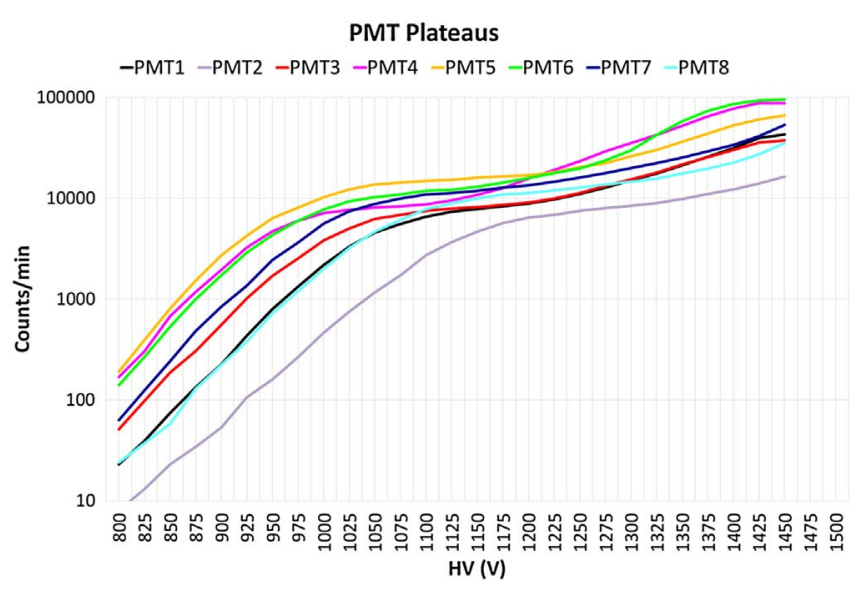

Fig. 15. PMT plateaus. The bias HV voltages were adjusted slightly above the flat section of the curves to obtain a more complete muon histogram.

Table 2. Pairings of PMTs and assigned bias voltage.

\begin{tabular}{lcccc}
\hline Photomultipliers & $\begin{array}{c}\text { PMT1- } \\
\text { PMT3 }\end{array}$ & $\begin{array}{c}\text { PMT2- } \\
\text { PMT8 }\end{array}$ & $\begin{array}{c}\text { PMT4- } \\
\text { PMT5 }\end{array}$ & $\begin{array}{c}\text { PMT6- } \\
\text { PMT7 }\end{array}$ \\
\hline Voltage & $1300 \mathrm{~V}$ & $1400 \mathrm{~V}$ & $1200 \mathrm{~V}$ & $1300 \mathrm{~V}$ \\
\hline
\end{tabular}

accomplished after telescope assembly by taking samples and generating histograms for each channel by means of a multi channel analizer (MCA). Then, the maximum of the histogram was brought to the same common value adjusting the gain controls placed in the printed circuit board for all the eight channels (Fig. 16).

As it is shown in the histogram on the right panel of Figure 16, hardly any pulses are received above level 4000 . For this reason, the ULD has been adjusted to level 6000 for all channels, rejecting high-level pulses which could saturate amplifier steps and generate false coincidences without interest in our application.

Eventually, the LLD adjustment was done by increasing the threshold of each channel until the number of counts was similar in all channels and slightly above the expected muon flux. In this way, particle detection is limited to those with the energy level in the expected range corresponding to that of muons.

\section{- Digital processing module adjustment}

As mentioned in Section 4.2, ARACNE doesn't need adjustments. All eight signals from the PMTs are sampled and recorded when a pulse is detected at any of the channels, and most of the LLD, ULD, coincidence selection and gain adjustments are performed later during data processing. Only a programmable trigger level needs to be programmed in the module.

Gain adjustment is performed by producing histograms with the recorded pulse height data for each channel, and aligning them through a correction (gain) factor, in the same way as it was done with the analog module (Fig. 16). The correction factor obtained for each channel is then used in equations (1)-(4) to calculate the coordinates as follows:

$$
N_{1}=L K_{1} V_{1} \text {. }
$$

Resulting equation (1) as follows:

$$
\begin{aligned}
& x_{T}=805.3 \ln \frac{L K_{3} V_{3}}{L K_{1} V_{1}}-0.0066 \Rightarrow \\
& x_{T}=805.3 \ln \frac{K_{3} V_{3}}{K_{1} V_{1}}-0.0066
\end{aligned}
$$

where $L$ is the relationship between the number of photons and the pulse height. $K_{1}$ and $K_{3}$ are the correction factor for channel 1 and 3. $V_{1}$ and $V_{3}$ the height pulses from PMT1 and PMT3 respectively.

The same gain correction process is applied to calculate the remaining coordinates. The correction factors obtained for this first MITO prototype are showed in Table 3.

Afterwards, ULD and LLD adjustments are carried out by filtering each channel data, taking only those that are below (ULD) and above (LLD) the appropriate thresholds, just as it was done with the analog module adjustment.

\section{Preliminary data}

MITO is currently operating at the Spanish Juan Carlos I Base at Livingston Island in Antarctica. Although it was installed in January 2019, more usable data have been produced since December 2019 when some calibration works were performed. Some examples of this preliminary data collected during January and February 2020 are presented below.

Four coincidence modes are activated during the normal operation time of MITO. Mode 1 (Coin8): coincidence of the eight PMTs. This mode implies that the detected particle crossed both scintillators, i. e. MITO-Top and MITO-Bottom. Mode 2 (Top): coincidence of the four PMTs of MITO-Top. Mode 3 (Bottom): coincidence of the four PMTs of MITOBottom and mode 4 (Diagonal): coincidence in PMTs number 1, 2, 7 and 8. This Diagonal mode serves as a test for other coincidence modes.

Data collected by MITO need some atmospheric corrections before being used for scientific purposes. Pressure and temperature effect on the measured counting rate must be corrected. It is generally accepted by many authors as (De Mendonça et al., 2013; Paschalis et al., 2013) among others, that the barometric effect can be experimentally determined by the following equation:

$$
\left(\frac{\Delta I}{I}\right)_{P}=\beta \cdot \Delta P
$$

where $(\Delta I / I)_{P}$ is the normalized deviation of the cosmic ray intensity in relation to atmospheric pressure, $\Delta P$ is the atmospheric pressure deviation and $\beta$ is the barometric coefficient, which depends on many factors, such as the nature of the secondary component and the altitude where the observation is performed (Dorman, 2004).

On the other hand, temperature corrections are more difficult although methods developed to describe and correct the effect of atmospheric temperature do exist. For instance, one of them 

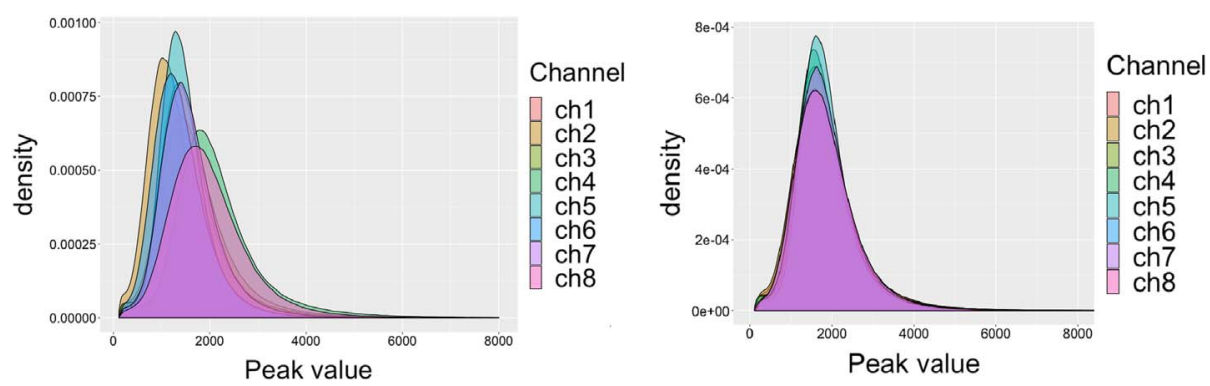

Fig. 16. Histograms with the 8 PMTs in coincidence, before and after the gain adjustment, but with LLD adjusted. They have the peak in the same value. After applying coincidence detection, noise disappears on the left of the histograms.

Table 3. Correction factors obtained for the gain adjustment of each channel.

\begin{tabular}{lcccccccc}
\hline Channel/PMT & 1 & 2 & 3 & 4 & 5 & 6 & 7 \\
\hline Correction factor & $K_{1}$ & $K_{2}$ & $K_{3}$ & $K_{4}$ & $K_{5}$ & $K_{6}$ & $K_{7}$ & $K_{8}$ \\
Value & 1.3 & 1.81 & 1.41 & 1 & 1.38 & 1.59 & 1.38 & 1.18 \\
\hline
\end{tabular}
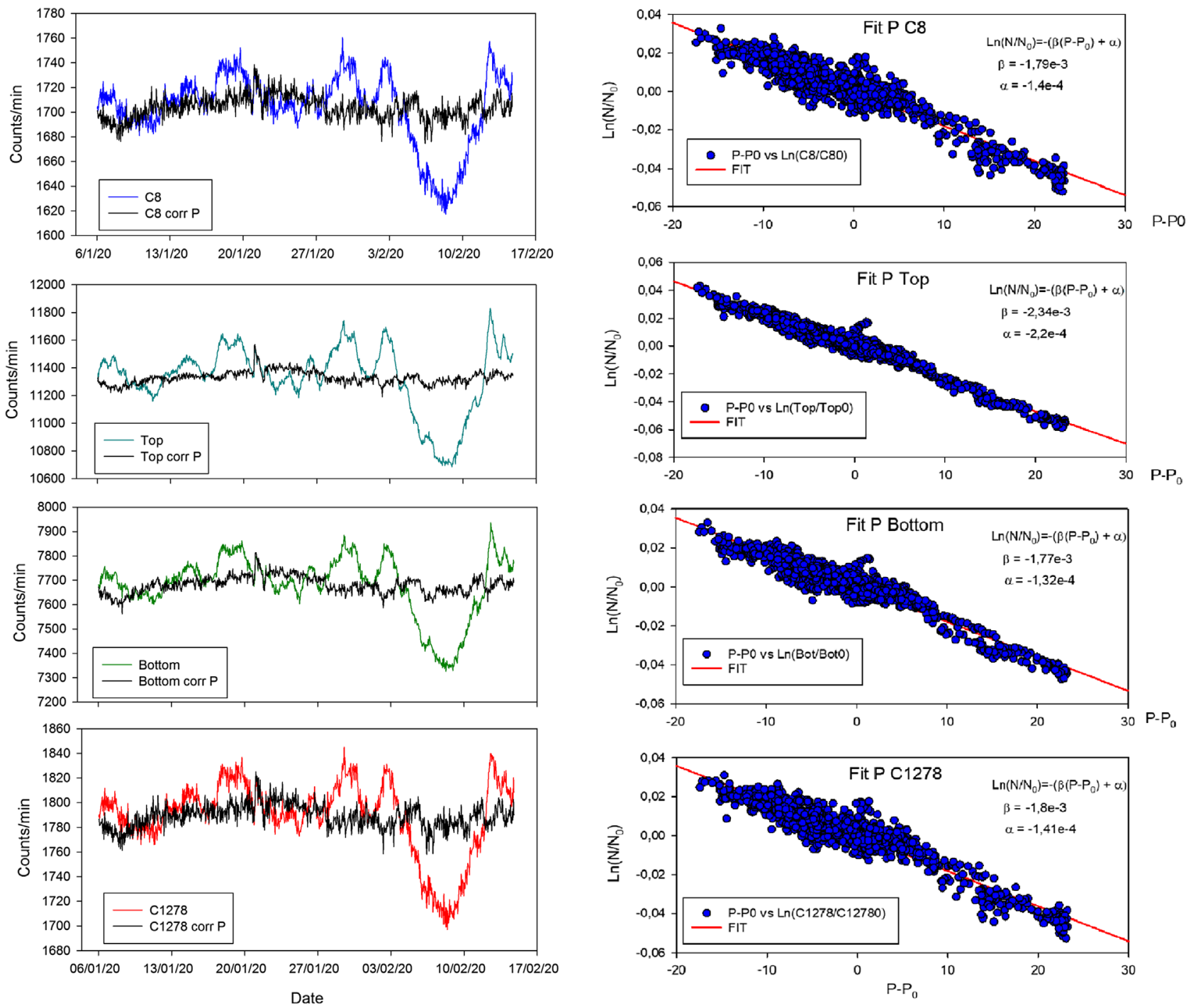

Fig. 17. On the left panel, the hourly count rate for data taken from January 6th to February 14th, 2020 for the four coincidence channels. Original signals (in color) and corrected pressure signals (in black). On the right, the four fitting plots to obtain the pressure adjusting parameters. 

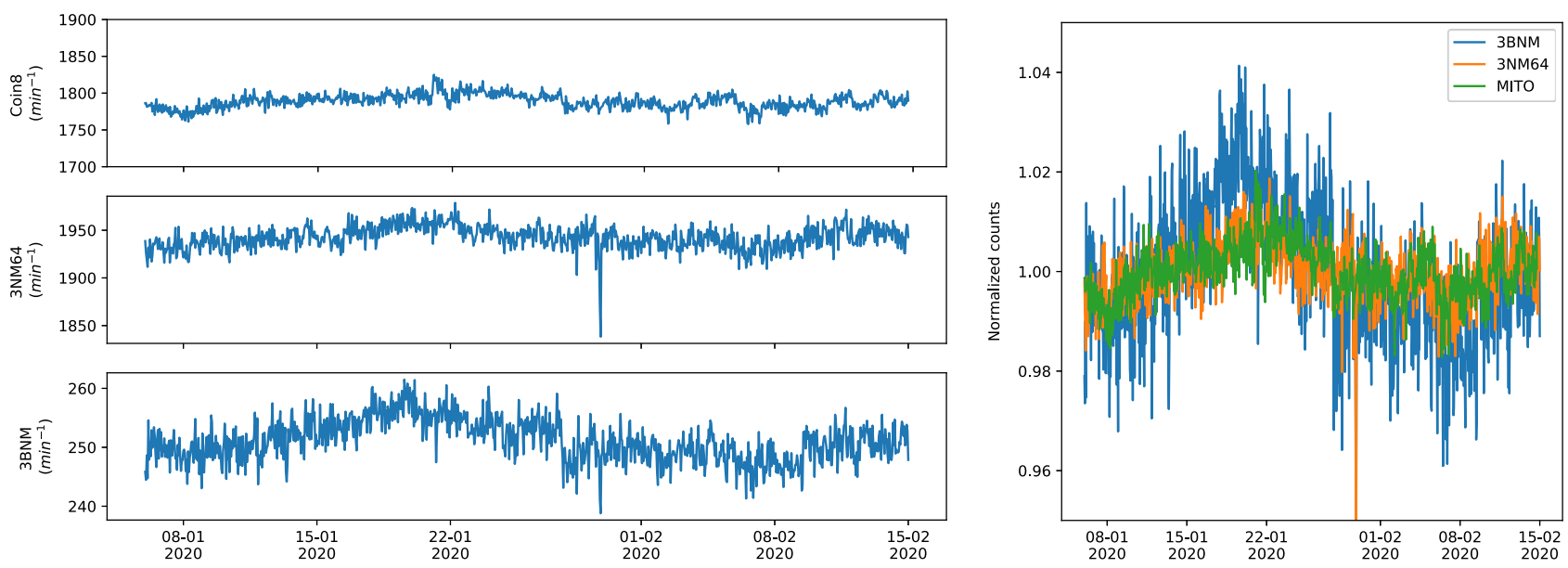

Fig. 18. On the left MITO (Coin8) and NEMO (3NM64 and 3BMN) fluxes with atmospheric pressure corrected from January 6th to February 14th, 2020. On the right panel the same normalized data. As can be seen, both MITO and NEMO fluxes present a clear correlation without great variations, which could be indicative of the absence of solar eruptive phenomena such as major solar flares or large CMEs.
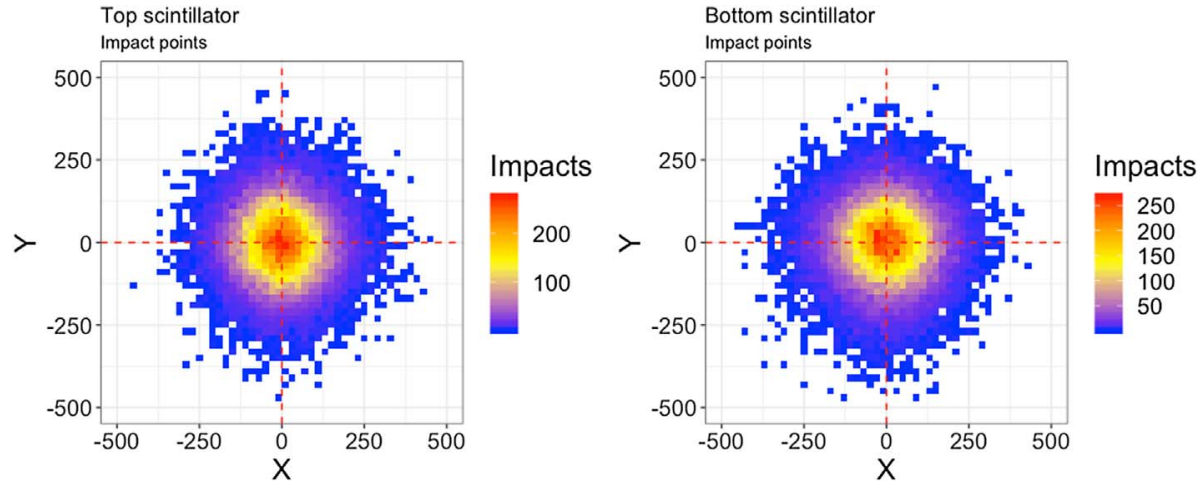

Fig. 19. Scatter plot of impact points in top and bottom scintillators with the eight PMTs in coincidence, corresponding to 34,783 data taken on January 31, 2019.

is the integral method which defines the normalized variation of muon flux dependence on temperature variation as:

$$
\left(\frac{\Delta I}{I}\right)_{T}=\int_{0}^{h_{0}} \alpha(h) \cdot \delta T(h) \cdot \mathrm{d} h
$$

where $\alpha(h)$ is the temperature coefficient density and $\delta T$ is the temperature variation calculated as $\delta T=T-T_{B}$, where $T$ is the actual temperature and $T_{B}$ is the base temperature value (Berkova et al., 2012).

The correction of the effect of temperature according the integral method (11) requires a complete information about the atmospheric temperature profile for a given geographical location and the temperature coefficient density function. Temperature profile measurements are often not available, as for Juan Carlos I Antarctic Base, and meteorological models have to be used instead (Berkova et al., 2012; Savic et al., 2016) have proven that meteorological models such as GFS (Global Forecast System) are able to provide temperatures for 25 isobaric levels for a given geographical location with latitude/longitude precision of $0.5^{\circ}$ what is suitable to replace the necessary temperature profile measurements for the estimation of the correction of the temperature effect on the MITO measurements.
Figure 17 represents the muon flux registered from January 6th to February 14th. Pressure corrected (black lines) and uncorrected (coloured lines) data are shown at the left column of the figure. Otherwise, the determination of the pressure coefficients for the four coincidence channel are presented at the right column of the figure. It is clear that the correction removes the main part of the atmospheric effect on MITO measurement.

A similar procedure to produce NEMO pressure-corrected data has been performed. MITO (muons) and NEMO (neutrons) can be directly compared. This is shown in Figure 18. As it can be seen, the flux is stable without great variations and very similar in both instruments, which could be in agreement with the fact that, in this period, there were no solar eruptive phenomena such as major flares and large CMEs.

Furthermore, from a technical point of view (main scope of this work), the flux is stable and the number of particles detected is within the expected margins. MITO-Top detects more impacts $\left(11,330 \mathrm{~min}^{-1}\right)$ than MITO-Bottom $\left(7680 \mathrm{~min}^{-1}\right)$, as it is expected due to the action of the intermediate lead layer. This behaviour is reproduced by the performed simulations. A stable flux is observed in all the coincidence channels with similar variations, which is indicative that the instrument is working properly. 
As it has been commented in previous sections, MITO can estimate the impact point of an incoming particle on the detector volume from the signal recorded by the photomultipliers. A set of 34,783 samples recorded along one hour on January 31st 2020 has been used to check this MITO capability. The computed impact points for MITO-Top (left) and MITO-bottom (right) are shown in Figure 19. Impact points have been computed using equations (1)-(4). Most of the impacts are in the central area of the scintillators as expected according to the telescope geometric factor and the muon angular distribution at sea level (Cecchini \& Spurio, 2012).

\section{Conclusions}

A new concept of directional muon telescope has been implemented and the first prototype is being tested at the Spanish Antarctic Base Juan Carlos I. Some of its functionalities, like muon flux counting and cosmic rays anisotropies determination, make it ideal to supply additional meaningful information to that provided by neutron monitors in Space Weather applications, anticipating the forecasts of ICMEs arriving on Earth and adding the ability to study additional characteristics of future incoming solar events.

The telescope has been equipped with two data acquisition modules that carry out similar functions using different technologies. Thus, the results from both systems can be compared and validated against each other. In addition, having a general purpose, yet powerful, single-board computer with networking capabilities, opens the possibility of making data available in real-time to the scientific community.

Compared to other large telescopes, MITO greatly reduces weight, volume and cost, achieving a good performance-cost ratio and therefore making it affordable. Although the point of impact calculation algorithms and some electronics may still be added to improve its performance and response in real-time, preliminary results seem to be reasonably in line with expectations.

Acknowledgements. We thank the following two projects: CTM2016-77325-C2-1-P, funded by Ministerio de Economía y Competitividad and by the European Regional Development Fund and PID2019-107806GB-I00, funded by Ministerio de Ciencia e Innovación. The editor thanks three anonymous reviewers for their assistance in evaluating this paper.

\section{References}

Aguayo Navarrete E, Kouzes RT, Ankney AS, Orrell JL, Berguson TJ, Troy MD. 2011. Cosmic ray interactions in shielding materials. https://doi.org/10.2172/1025678, https://www.pnnl.gov/ main/491publications/external/technical_reports/PNNL-20693.pdf.

Ayuso S, Blanco JJ, Medina J, Gómez-Herrero R, García-Población O, García Tejedor I. 2016. A coincidence detection system based on real-time software. Geosci Instrum Methods Data Syst 5(2): 437-449. https://doi.org/10.5194/gi-5-437-2016, URL https://gi. copernicus.org/articles/5/437/2016/.
Ayuso S, García Tejedor JI, Blanco JJ, Gómez Herrero R, García Población O, Medina J, Prieto M, López Comazzi A. 2019. MITO, a new directional muon telescope design. First observations. PoS ICRC2019: 180. https://doi.org/10.22323/1.358.0180, URL https://pos.sissa.it/358/180/.

Berkova M, Belov A, Eroshenko E, Yanke V. 2012. Temperature effect of muon component and practical questions of how to take into account in real time. Astrophys Space Sci Trans 8(1): 41-44. https://doi.org/10.5194/astra-8-41-2012, URL http://www.astrophys-space-sci-trans.net/8/41/2012/.

Blanco JJ, Catalán E, Hidalgo MA, Medina J, García O, RodríguezPacheco J. 2013a. Observable effects of interplanetary coronal mass ejections on ground level neutron monitor count rates. Sol Phys 284(1): 167-178. https://doi.org/10.1007/s11207-013-0256-1, URL https://link.springer.com/article/10.1007/s11207-013-0256-1.

Blanco JJ, Hidalgo MA, Gómez-Herrero R, Rodríguez-Pacheco J, Heber B, Wimmer-Schweingruber RF, Martín C. 2013b. Energetic-particle-flux decreases related to magnetic cloud passages as observed by the Helios 1 and 2 spacecraft. A\&A 556: A146. https://doi.org/10.1051/0004-6361/201321739.

Blanco JJ, García Población O, García Tejedor JI, Medina J, Prieto M, López-Comazzi A, Ayuso S, Gómez-Herrero R, Steigies C. 2019. A new neutron monitor at the Juan Carlos I Spanish Antarctic Station (Livingston Island-Antarctic Peninsula). PoS ICRC2019: 1060. https://doi.org/10.22323/1.358.1060, URL https://pos.sissa.it/358/1060/.

Cane HV. 2000. Coronal mass ejections and forbush decreases. Space Sci Rev 93: 55-77. https://doi.org/10.1023/A:1026532125747.

Cane HV, Richardson IG, St. Cyr OC. 2000. Coronal mass ejections, interplanetary ejecta and geomagnetic storms. Geophys Res Lett 27(21): 3591-3594. https://doi.org/10.1029/2000GL000111, URL https://agupubs.onlinelibrary.wiley.com/doi/abs/10.1029/ 2000GL000111.

Cecchini S, Spurio M. 2012. Atmospheric muons: experimental aspects. Geosci Instrum Methods Data Syst 1(2): 185-196. https://doi.org/10.5194/gi-1-185-2012, URL https://www.geosciinstrum-method-data-syst.net/1/185/2012/.

da Silva MR, Contreira DB, Monteiro S, Trivedi NB, Munakata K, Kuwabara T, Schuch NJ. 2004. Cosmic ray muon observation at southern space observatory - SSO (29S, 53W). Astrophys Space Sci 290(3): 389-397. https://doi.org/10.1023/B:ASTR. 0000032537.23712.22, URL https://ui.adsabs.harvard.edu/abs/ 2004Ap\&SS.290.389D.

De Mendonça RRS, Raulin JP, Echer E, Makhmutov VS, Fernandez G. 2013. Analysis of atmospheric pressure and temperature effects on cosmic ray measurements. J Geophys Res: Space Phys 118(4): 1403-1409. https://doi.org/10.1029/2012JA018026, URL https:// agupubs.onlinelibrary.wiley.com/doi/abs/10.1029/2012JA018026.

Dorman L. 2004. Cosmic rays in the Earth's atmosphere and underground, Vol. 303. https://doi.org/10.1007/978-1-4020-2113-8, URL https://link.springer.com/book/10.1007\%2F978-1-4020-2113-8.

Duldig M. 2000. Muon observations. Space Sci Rev 93: 207-226. https://doi.org/10.1023/A:1026596529381, URL https:// link.springer.com/article/10.1023\%2FA\%3A1026596529381.

Havelka M, Auerbach P, Sochorová J. 2002. Software coincidence counting. Appl Radiat Isot 56(1): 265-268. Proceedings of the Conference on Radionuclide Metrology and its Applications, ICRM'01. https://doi.org/10.1016/S0969-8043(01)00198-1, URL http://www.sciencedirect.com/science/article/pii/S096980430100 1981.

Karapetyan Grigori, Ganeva Marina, Hippler Rainer. 2013. Directional sensitivity of MuSTAnG muon telescope. J Space Weather Space Clim 3: A16. https://doi.org/10.1051/swsc/2013040. 
Leerungnavarat K, Ruffolo D, Bieber JW. 2003. Loss cone precursors to forbush decreases and advance warning of space weather effects. Astrophys J 593(1): 587-596. https://doi.org/10.1086/376408, https://iopscience.iop.org/article/10.1086/376408.

Mavromichalaki H, Yanke V, Dorman L, Iucci N, Chilingaryan A, Kryakunova O. 2004. Neutron Monitor Network In Real Time And Space Weather. In: Effects of Space Weather on Technology Infrastructure, Daglis IA (Ed.), Springer, Netherlands, Dordrecht, pp. 301-317. ISBN 978-1-4020-2754-3. https://doi.org/10.1007/ 1-4020-2754-0_16, http://fzk.aragats.am/files/Publication/Neutron_ Monitor_Network_in_Real_Time_and_Space_Wea.pdf, URL https:// link.springer.com/chapter/10.1007\%2F1-4020-2754-0_16.

Medina J, Blanco JJ, García O, Gómez-Herrero R, Catalán EJ, et al. 2013. Castilla-La Mancha neutron monitor. Nucl Instrum Methods Phys Res Sect A: Accel Spectr Detect Assoc Equip 727: 97-103. https://doi.org/10.1016/j.nima.2013.06.028, http://www.sciencedirect. com/science/article/pii/S0168900213008486.

Mendonça RRS, Wang C, Braga CR, Echer E, Dal Lago A, et al. 2019. Analysis of cosmic rays' atmospheric effects and their relationships to cutoff rigidity and zenith angle using global muon detector network Data. J Geophys Res: Space Phys 124(12): 9791-9813. https://doi.org/10.1029/2019JA026651, https://agupubs.onlinelibrary. wiley.com/doi/pdf/10.1029/2019JA026651, https://agupubs. onlinelibrary.wiley.com/doi/abs/10.1029/2019JA026651.

Müller-Mellin R, Kunow H, Fleißner V, Pehlke E, Rode E, et al. 1995. COSTEP - comprehensive suprathermal and energetic particle analyser. Sol Phys 162(1-2): 483-504. https://doi.org/10.1007/ BF00733437, http://articles.adsabs.harvard.edu/pdf/1995SoPh.162. 483.

Munakata K, Bieber JW, Yasue S-I, Kato C, Koyama M, Akahane S, Fujimoto K, Fujii Z, Humble JE, Duldig ML. 2000. Precursors of geomagnetic storms observed by the muon detector network. J Geophys Res: Space Phys 105(A12): 27457-27468. https://doi. org/10.1029/2000JA000064, https://agupubs.onlinelibrary.wiley. com/doi/pdf/10.1029/2000JA000064, https://agupubs.onlinelibrary. wiley.com/doi/abs/10.1029/2000JA000064.

Munakata K, Kuwabara T, Yasue S, Kato C, Akahane S, et al. 2005. A "loss cone" precursor of an approaching shock observed by a cosmic ray muon hodoscope on October 28, 2003. Geophys Res Lett 32(3): L03S04 (1-4). https://doi.org/10.1029/2004GL021469, https://agupubs.onlinelibrary.wiley.com/doi/pdf/10.1029/ 2004GL021469, https://agupubs.onlinelibrary.wiley.com/doi/abs/ 10.1029/2004GL021469, https://agupubs.onlinelibrary.wiley.com/ action/showCitFormats?doi=10.1029\%2F2004GL021469.
Munakata K, Kozai M, Evenson P, Kuwabara T, Kato C, et al. 2018. Cosmic-ray short burst observed with the Global Muon Detector Network (GMDN) on 2015 June 22. Astrophys J 862(2): 170. https://doi.org/10.3847/1538-4357/aacdfe, URL https://doi.org/ $10.3847 \%$ F F1538-4357\%2Faacdfe.

Paschalis P, Mavromichalaki H, Yanke V, Belov A, Eroshenko E, Gerontidou M, Koutroumpi I. 2013. Online application for the barometric coefficient calculation of the NMDB stations. New Astron 19: 10-18. https://doi.org/10.1016/j.newast.2012.08.003, URL http:// www.sciencedirect.com/science/article/pii/S1384107612000759.

Pharr M, Jakob W, Humphreys G. 2017. 08 - Reflection models. In: Physically based rendering (third edition), Pharr M, Jakob W, Humphreys G (Eds.), third edn, Morgan Kaufmann, Boston, pp. 507-568. ISBN 978-0-12-800645-0. https://doi.org/10.1016/ B978-0-12-800645-0.50008-7, URL http://www.sciencedirect.com/ science/article/pii/B9780128006450500087.

Regadío A, Tejedor JIG, Ayuso S, Población Óscar García, Blanco JJ, Sánchez-Prieto S, Polo Óscar Rodriguez. 2020. Trajectory determination of muons using scintillators and a novel selforganizative map. Nucl Instrum Methods Phys Res Sect A: Accel Spectr Detect Assoc Equip 973: 164-166. https://doi.org/10.1016/ j.nima.2020.164166, URL http://www.sciencedirect.com/science/ article/pii/S0168900220305623.

Rockenbach M, Dal Lago A, Schuch N, Munakata K, Kuwabara T, et al. 2014. Global muon detector network used for space weather applications. Space Sci Rev 182(1-4): 1-18. URL https://link. springer.com/article/10.1007\%2Fs11214-014-0048-4.

Sato T, Yasuda H, Niita K, Endo A, Sihver L. 2008. Development of PARMA: PHITS-based analytical radiation model in the atmosphere. Radiat Res 170(2): 244-259. https://doi.org/10.1667/ RR1094.1, URL https://meridian.allenpress.com/radiation-research/ article-pdf/170/2/244/2155866/rr1094_1.pdf.

Savic M, Dragic A, Veselinovic N, Udovicic V, Banjanac R, Jokovic D, Maletic D. 2016. Effect of pressure and temperature corrections on muon flux variability at ground level and underground. https:// arxiv.org/pdf/1701.00164.pdf.

Tanabashi M, Hagiwara K, Hikasa K, Nakamura K, Sumino Y, et al (Particle Data Group). 2018. Review of particle physics. Phys Rev D 98: 030,001. https://doi.org/10.1103/PhysRevD.98.030001, URL https://link.aps.org/doi/10.1103/PhysRevD.98.030001.

Wright AG. 2017. The photomultiplier handbook. Oxford University Press, Oxford. https://doi.org/10.1093/oso/9780199565092.001.0001, URL https://cds.cern.ch/record/2258194.

Cite this article as: Ayuso S, Blanco JJ, Tejedor JIG, Herrero RG, Vrublevskyy I, et al. 2021. MITO: a new directional muon telescope. J. Space Weather Space Clim. 11, 13. https://doi.org/10.1051/swsc/2020079. 